TRANSACTIONS OF THE

AMERICAN MATHEMATICAL SOCIETY

Volume 355, Number 1, Pages 273-314

S 0002-9947(02)03096-9

Article electronically published on August 7, 2002

\title{
MATRIX-WEIGHTED BESOV SPACES
}

\author{
SVETLANA ROUDENKO
}

\begin{abstract}
Nazarov, Treil and Volberg defined matrix $A_{p}$ weights and extended the theory of weighted norm inequalities on $L^{p}$ to the case of vectorvalued functions. We develop some aspects of Littlewood-Paley function space theory in the matrix weight setting. In particular, we introduce matrixweighted homogeneous Besov spaces $\dot{B}_{p}^{\alpha q}(W)$ and matrix-weighted sequence Besov spaces $\dot{b}_{p}^{\alpha q}(W)$, as well as $\dot{b}_{p}^{\alpha q}\left(\left\{A_{Q}\right\}\right)$, where the $A_{Q}$ are reducing operators for $W$. Under any of three different conditions on the weight $W$, we prove the norm equivalences $\|\vec{f}\|_{\dot{B}_{p}^{\alpha q}(W)} \approx\left\|\left\{\vec{s}_{Q}\right\}_{Q}\right\|_{\dot{b}_{p}^{\alpha q}(W)} \approx\left\|\left\{\vec{s}_{Q}\right\}_{Q}\right\|_{\dot{b}_{p}^{\alpha q}\left(\left\{A_{Q}\right\}\right)}$, where $\left\{\vec{s}_{Q}\right\}_{Q}$ is the vector-valued sequence of $\varphi$-transform coefficients of $\vec{f}$. In the process, we note and use an alternate, more explicit characterization of the matrix $A_{p}$ class. Furthermore, we introduce a weighted version of almost diagonality and prove that an almost diagonal matrix is bounded on $\dot{b}_{p}^{\alpha q}(W)$ if $W$ is doubling. We also obtain the boundedness of almost diagonal operators on $\dot{B}_{p}^{\alpha q}(W)$ under any of the three conditions on $W$. This leads to the boundedness of convolution and non-convolution type Calderón-Zygmund operators $(\mathrm{CZOs})$ on $\dot{B}_{p}^{\alpha q}(W)$, in particular, the Hilbert transform. We apply these results to wavelets to show that the above norm equivalence holds if the $\varphi$-transform coefficients are replaced by the wavelet coefficients. Finally, we construct inhomogeneous matrix-weighted Besov spaces $B_{p}^{\alpha q}(W)$ and show that results corresponding to those above are true also for the inhomogeneous case.
\end{abstract}

\section{Introduction. Overview of the Results}

Littlewood-Paley theory gives a unified perspective to the theory of function spaces. Well-known spaces such as Lebesgue, Hardy, Sobolev, Lipschitz spaces, etc. are special cases of either Besov spaces $\dot{B}_{p}^{\alpha q}$ (homogeneous), $B_{p}^{\alpha q}$ (nonhomogeneous) or Triebel-Lizorkin spaces $\dot{F}_{p}^{\alpha q}$ (homogeneous), $F_{p}^{\alpha q}$ (nonhomogeneous) (e.g., see [15]). The properties of these spaces are characterized by their discrete analogues: the sequence Besov spaces $\dot{b}_{p}^{\alpha q}, b_{p}^{\alpha q}$ and sequence Triebel-Lizorkin spaces $\dot{f}_{p}^{\alpha q}, f_{p}^{\alpha q}([4],[3])$. Littlewood-Paley theory provides alternate methods for studying singular integrals. The Hilbert transform, the classical example of a singular integral operator, led to the extensive modern theory of Calderón-Zygmund operators, mostly studied on the Lebesgue $L^{p}$ spaces.

Motivated by the fundamental result of M. Riesz in the 1920s that the Hilbert transform preserves $L^{p}$ for $1<p<\infty$, Hunt, Muckenhoupt and Wheeden showed

Received by the editors March 15, 2002.

2000 Mathematics Subject Classification. Primary 42B25, 42B35, 47B37, 47B38.

Key words and phrases. Besov spaces, matrix weights, $\varphi$-transform, $A_{p}$ condition, doubling measure, reducing operators, almost diagonal operators, Calderón-Zygmund operators, Hilbert transform, wavelets. 
that the famous $A_{p}$ condition on a weight $w$ is the necessary and sufficient condition for the Hilbert transform to be bounded on $L^{p}(w)(1973,[8])$. More recent developments deal with matrix-weighted spaces where scalar methods simply could not be applied. In 1996 Treil and Volberg obtained the analogue of the HuntMuckenhoupt-Wheeden condition for the matrix case when $p=2(16])$. Soon afterwards, Nazarov and Treil introduced in [12] a new "Bellman function" method to extend the theory to $1<p<\infty$. In 1997 Volberg presented a different solution to the matrix weighted $L^{p}$ boundedness of the Hilbert transform via techniques related to classical Littlewood-Paley theory ([17]).

The purpose of this paper is to extend some aspects of Littlewood-Paley function space theory, in particular, the study of Besov spaces and Calderón-Zygmund operators on them, previously obtained with no weights and partially for scalar weights, to the matrix weight setting.

We define a new generalized function space: the vector-valued homogeneous Besov space $\dot{B}_{p}^{\alpha q}(W)$ with matrix weight $W$. Let $\mathcal{M}$ be the cone of nonnegative definite operators on a Hilbert space $\mathcal{H}$ of dimension $m$ (consider $\mathcal{H}=\mathbb{C}^{m}$ or $\left.\mathbb{R}^{m}\right)$, i.e., for $M \in \mathcal{M}$ we have $(M x, x)_{\mathcal{H}} \geq 0$ for all $x \in \mathcal{H}$. By definition, a matrix weight $W$ is an a.e. invertible map $W: \mathbb{R}^{n} \rightarrow \mathcal{M}$. For a measurable $\vec{g}=\left(g_{1}, \ldots, g_{m}\right)^{\mathrm{T}}: \mathbb{R}^{n} \rightarrow \mathcal{H}$, let $\|\vec{g}\|_{L^{p}(W)}=\left(\int_{\mathbb{R}^{n}}\left\|W^{1 / p}(t) \vec{g}(t)\right\|_{\mathcal{H}}^{p} d t\right)^{1 / p}$. If the previous norm is finite, then $\vec{g} \in L^{p}(W)$. We say that a function $\varphi \in \mathcal{S}\left(\mathbb{R}^{n}\right)$ belongs to the class $\mathcal{A}$ of admissible kernels if supp $\hat{\varphi} \subseteq\left\{\xi \in \mathbb{R}^{n}: \frac{1}{2} \leq|\xi| \leq 2\right\}$ and $|\hat{\varphi}(\xi)| \geq c>0$ if $\frac{3}{5} \leq|\xi| \leq \frac{5}{3}$. Set $\varphi_{\nu}(x)=2^{\nu n} \varphi\left(2^{\nu} x\right)$ for $\nu \in \mathbb{Z}$.

Definition 1.1 (Matrix-weighted Besov space $\dot{B}_{p}^{\alpha q}(W)$ ). For $\alpha \in \mathbb{R}, 1 \leq p<\infty$, $0<q \leq \infty, \varphi \in \mathcal{A}$ and $W$ a matrix weight, the Besov space $\dot{B}_{p}^{\alpha q}(W)$ is the collection of all vector-valued distributions $\vec{f}=\left(f_{1}, \ldots, f_{m}\right)^{\mathrm{T}}$ with $f_{i} \in \mathcal{S}^{\prime} / \mathcal{P}\left(\mathbb{R}^{n}\right), 1 \leq i \leq m$ (the space of tempered distributions modulo polynomials) such that

$$
\|\vec{f}\|_{\dot{B}_{p}^{\alpha q}(W)}=\left\|\left\{2^{\nu \alpha}\left\|\varphi_{\nu} * \vec{f}\right\|_{L^{p}(W)}\right\}_{\nu}\right\|_{l^{q}}=\left\|\left\{\left\|W^{1 / p} \cdot\left(\varphi_{\nu} * \vec{f}\right)\right\|_{L^{p}}\right\}_{\nu}\right\|_{l_{q}^{\alpha}}<\infty
$$

where $\varphi_{\nu} * \vec{f}=\left(\varphi_{\nu} * f_{1}, \ldots, \varphi_{\nu} * f_{m}\right)^{\mathrm{T}}$ and the $l^{q}$-norm is replaced by the supremum on $\nu$ if $q=\infty$.

The case $p=\infty$ is not of interest to us, since $\dot{B}_{\infty}^{\alpha q}(W)=\dot{B}_{\infty}^{\alpha q}$ because $L^{\infty}(W)=$ $L^{\infty}$. Since $\varphi$ is directly involved in the definition of $\dot{B}_{p}^{\alpha q}(W)$, there seems to be a dependence on the choice of $\varphi: \dot{B}_{p}^{\alpha q}(W)=\dot{B}_{p}^{\alpha q}(W, \varphi)$. Under appropriate conditions on $W$, Theorem 1.8 below shows that this is not the case. The space $\dot{B}_{p}^{\alpha q}(W)$ is complete, as is discussed at the end of Section 7 .

We also introduce the corresponding weighted sequence (discrete) Besov space $\dot{b}_{p}^{\alpha q}(W)$ :

Definition 1.2 (Matrix-weighted sequence Besov space $\dot{b}_{p}^{\alpha q}(W)$ ). For $\alpha \in \mathbb{R}, 1 \leq$ $p<\infty, 0<q \leq \infty$ and $W$ a matrix weight, the space $\dot{b}_{p}^{\alpha q}(W)$ consists of all vector-valued sequences $\vec{s}=\left\{\vec{s}_{Q}\right\}_{Q}$, where $\vec{s}_{Q}=\left(s_{Q}^{(1)}, \ldots, s_{Q}^{(m)}\right)^{\mathrm{T}}$, enumerated by 
the dyadic cubes $Q$ contained in $\mathbb{R}^{n}$, such that

$$
\begin{aligned}
& \left\|\left\{\vec{s}_{Q}\right\}_{Q}\right\|_{b_{p}^{\alpha q}(W)}=\left\|\left\{2^{\nu \alpha}\left\|\sum_{l(Q)=2^{-\nu}}|Q|^{-\frac{1}{2}} \vec{s}_{Q} \chi_{Q}\right\|_{L^{p}(W)}\right\}_{\nu}\right\|_{l^{q}} \\
= & \left\|\left\{\left\|\sum_{l(Q)=2^{-\nu}}|Q|^{-\frac{1}{2}}\left(\left\|W^{1 / p}(t) \vec{s}_{Q}\right\|_{\mathcal{H}}\right) \chi_{Q}(t)\right\|_{L^{p}(d t)}\right\}_{\nu}\right\|_{l_{q}^{\alpha}}<\infty,
\end{aligned}
$$

where $|Q|$ is the Lebesgue measure of $Q, l(Q)$ is the side length of $Q$, and the $l^{q}$-norm is again replaced by the supremum on $\nu$ if $q=\infty$.

For $\nu \in \mathbb{Z}$ and $k \in \mathbb{Z}^{n}$, let $Q_{\nu k}$ be the dyadic cube $\left\{\left(x_{1}, \ldots, x_{n}\right) \in \mathbb{R}^{n}: k_{i} \leq\right.$ $\left.2^{\nu} x_{i}<k_{i}+1, i=1, \ldots, n\right\}$, and $x_{Q}=2^{-\nu} k$ the lower left corner of $Q_{\nu k}$. Set $\varphi_{Q}(x)=|Q|^{-1 / 2} \varphi\left(2^{\nu} x-k\right)=|Q|^{1 / 2} \varphi_{\nu}\left(x-x_{Q}\right)$ for $Q=Q_{\nu k}$. For each $\vec{f}$ with $f_{i} \in \mathcal{S}^{\prime}\left(\mathbb{R}^{n}\right)$, we define the $\varphi$-transform $S_{\varphi}$ as the map taking $\vec{f}$ to the vector-valued sequence $S_{\varphi}(\vec{f})=\left\{\left\langle\vec{f}, \varphi_{Q}\right\rangle\right\}_{Q}=\left\{\left(\left\langle f_{1}, \varphi_{Q}\right\rangle, \ldots,\left\langle f_{m}, \varphi_{Q}\right\rangle\right)^{\mathrm{T}}\right\}_{Q}$ for $Q$ dyadic. We call $\vec{s}_{Q}(\vec{f}):=\left\langle\vec{f}, \varphi_{Q}\right\rangle$ the $\varphi$-transform coefficients of $\vec{f}$.

The next question is motivated by the following results:

(i) Frazier and Jawerth $([4], 1985)$ showed that, in the unweighted scalar case,

$$
\|f\|_{\dot{B}_{p}^{\alpha q}} \approx\left\|\left\{s_{Q}(f)\right\}_{Q}\right\|_{\dot{b}_{p}^{\alpha q}},
$$

where $\left\{s_{Q}(f)\right\}_{Q}$ are the $\varphi$-transform coefficients. A similar equivalence holds if $\left\{s_{Q}(f)\right\}_{Q}$ are the wavelet coefficients $\left\{\left\langle f, \psi_{Q}\right\rangle\right\}_{Q}$ of $f$ with $\psi_{Q}$ being smooth, say, Meyer's wavelets (ref. [11).

(ii) Nazarov, Treil and Volberg ([12], 1996, [17], 1997) obtained

$$
\|\vec{f}\|_{L^{p}(W)} \approx\left\|\left\{\left\langle\vec{f}, h_{Q}\right\rangle\right\}\right\|_{f_{p}^{02}(W)} \quad \text { if } W \in A_{p},
$$

where $\left\{h_{Q}\right\}_{Q}$ is the Haar system and $\dot{f}_{p}^{02}(W)$ is the coefficient (sequence Triebel-Lizorkin) space for $L^{p}(W)$. A particular case of (1.1), when $m=1$ and $w$ is a scalar weight, is

$$
\|f\|_{\dot{B}_{2}^{02}(w)}=\|f\|_{L^{2}(w)} \approx\left\|\left\{\left\langle f, h_{Q}\right\rangle\right\}\right\|_{\dot{f}_{2}^{02}(w)}=\left\|\left\{\left\langle f, h_{Q}\right\rangle\right\}\right\|_{\dot{b}_{2}^{02}(w)},
$$

where the first equality and the equivalence hold if $w \in A_{2}$.

For our purposes we will use a condition on $W$ that is equivalent to the matrix $A_{p}$ condition of [12] (for the proof, refer to Section 3):

Lemma 1.3. Let $W$ be a matrix weight, $1<p<\infty$, and let $p^{\prime}$ be the conjugate of $p\left(\frac{1}{p}+\frac{1}{p^{\prime}}=1\right)$. Then

$$
\int_{B}\left(\int_{B}\left\|W^{1 / p}(x) W^{-1 / p}(t)\right\|^{p^{\prime}} \frac{d t}{|B|}\right)^{p / p^{\prime}} \frac{d x}{|B|} \leq c_{p, n} \text { for every ball } B \subseteq \mathbb{R}^{n}
$$

if and only if $W \in A_{p}$. 
In (1.2), $\left\|W^{1 / p}(x) W^{-1 / p}(t)\right\|$ refers to the matrix (operator) norm.

The advantage of condition (1.2) is that it allows us to understand the $A_{p}$ condition in terms of matrices, avoiding metrics $\rho, \rho^{*}$ and their averagings as well as reducing operators (for definitions and details refer to Section 3 ).

Our first result is the norm equivalence between the continuous matrix-weighted Besov space $\dot{B}_{p}^{\alpha q}(W)$ and the discrete matrix-weighted Besov space $\dot{b}_{p}^{\alpha q}(W)$ under the $A_{p}$ condition:

Theorem 1.4. Let $\alpha \in \mathbb{R}, 0<q \leq \infty, 1<p<\infty$ and $W \in A_{p}$. Then

$$
\|\vec{f}\|_{\dot{B}_{p}^{\alpha q}(W)} \approx\left\|\left\{\vec{s}_{Q}(\vec{f})\right\}_{Q}\right\|_{\dot{b}_{p}^{\alpha q}(W)} .
$$

In some cases, the $A_{p}$ requirement on $W$ can be relaxed. Recall that a scalar measure $\mu$ is called doubling if there exists $c>0$ such that for any $\delta>0$ and any $z \in \mathbb{R}^{n}$,

$$
\mu\left(B_{2 \delta}(z)\right) \leq c \mu\left(B_{\delta}(z)\right),
$$

where $B_{\delta}(z)=\left\{x \in \mathbb{R}^{n}:|z-x|<\delta\right\}$.

Definition 1.5 (Doubling matrix). A matrix weight $W$ is called a doubling matrix (of order $p, 1 \leq p<\infty$ ), if there exists a constant $c=c_{p, n}$ such that for any $y \in \mathcal{H}$, any $\delta>0$ and any $z \in \mathbb{R}^{n}$,

$$
\int_{B_{2 \delta}(z)}\left\|W^{1 / p}(t) y\right\|_{\mathcal{H}}^{p} d t \leq c \int_{B_{\delta}(z)}\left\|W^{1 / p}(t) y\right\|_{\mathcal{H}}^{p} d t
$$

i.e., the scalar measure $w_{y}(t)=\left\|W^{1 / p}(t) y\right\|_{\mathcal{H}}^{p}$ is uniformly doubling and not identically zero (a.e.). If $c=2^{\beta}$ is the smallest constant for which (1.5) holds, then $\beta$ is called the doubling exponent of $W$.

It is known that if $W \in A_{p}$, then $w_{y}$ is a scalar $A_{p}$ weight for any $y \in \mathcal{H}$ and the $A_{p}$ constant is independent of $y$ (for example, see [17]). This, in turn, implies that $w_{y}$ is a scalar doubling measure (e.g., see [14]) and the doubling constant is also independent of $y$. Using decomposition techniques, we prove the equivalence (1.3) under the doubling assumption on $W$ with the restriction that $p$ is large, and with no restriction on $p$ in the case when $W$ is a diagonal matrix:

Theorem 1.6. Let $\alpha \in \mathbb{R}, 0<q \leq \infty, 1 \leq p<\infty$, and let $W$ be a doubling matrix of order $p$ with doubling exponent $\beta$. Suppose $p>\beta$. Then the norm equivalence (1.3) holds. If $W$ is diagonal, then (1.3) holds for all $1 \leq p<\infty$.

The case of a scalar weight is a particular case of the diagonal matrix weight case, and thus, the equivalence (1.3) holds just under the doubling condition. This fact is essentially known (see [5] for the case of $\dot{F}_{p}^{\alpha q}$ ); it is proved here for purposes of comparison and generalization to the diagonal matrix case.

Remark 1.7. One of the directions of the norm equivalence uses only the doubling property of $W$ with no restrictions (see Corollary [5.6), but the other direction requires the stated assumptions on $W$ (see Theorem 6.6). Furthermore, the first direction is obtained from a more general norm estimate involving families of "smooth molecules" (see Theorem 5.2).

Summarizing Theorems 1.4 and 1.6, the norm equivalence (1.3) holds under any of the following conditions: 
(A1) $W \in A_{p}$ with $1 \leq p<\infty$,

(A2) $W$ is a doubling matrix of order $p$ with $p>\beta$, where $\beta$ is the doubling exponent of $W$

(A3) $W$ is a diagonal doubling matrix of order $p$ with $1 \leq p<\infty$.

Now we will state the independence of the space $\dot{B}_{p}^{\alpha q}(W, \varphi)$ from $\varphi$ :

Theorem 1.8. Let $\vec{f} \in \dot{B}_{p}^{\alpha q}\left(W, \varphi^{(1)}\right), \varphi^{(1)} \in \mathcal{A}, \alpha \in \mathbb{R}, 0<q \leq \infty, 1 \leq p<\infty$, and suppose any of (A1)-(A3) hold. Then for any $\varphi^{(2)} \in \mathcal{A}$,

$$
\|\vec{f}\|_{\dot{B}_{p}^{\alpha q}\left(W, \varphi^{(1)}\right)} \approx\|\vec{f}\|_{\dot{B}_{p}^{\alpha q}\left(W, \varphi^{(2)}\right)} .
$$

If we use the language of reducing operators (see [17 or Section 3 below), we extend the norm equivalence (1.3) to a different sequence space, namely $\dot{b}_{p}^{\alpha q}\left(\left\{A_{Q}\right\}\right)$. For each dyadic cube $Q$, consider a reducing operator $A_{Q}$ corresponding to the $L^{p}$ average over $Q$ of the norm $\left\|W^{1 / p} \cdot\right\|_{\mathcal{H}}$, i.e.,

$$
\left\|A_{Q} \vec{u}\right\|_{\mathcal{H}} \approx\left(\frac{1}{|Q|} \int_{Q}\left\|W^{1 / p}(t) \vec{u}\right\|_{\mathcal{H}}^{p} d t\right)^{1 / p}
$$

for all vector-valued sequences $\vec{u}$. Note that the assumption that $W$ is a.e. invertible guarantees that each $A_{Q}$ is invertible. Define the sequence space $\dot{b}_{p}^{\alpha q}\left(\left\{A_{Q}\right\}\right)$ for $\alpha \in \mathbb{R}, 1 \leq p<\infty, 0<q \leq \infty$ as the space containing all vector-valued sequences $\left\{\vec{s}_{Q}\right\}_{Q}$ with

$$
\left\|\left\{\vec{s}_{Q}\right\}_{Q}\right\|_{\dot{b}_{p}^{\alpha q}\left(\left\{A_{Q}\right\}\right)}=\left\|\left\{2^{\nu \alpha}\left\|\sum_{l(Q)=2^{-\nu}}|Q|^{-\frac{1}{2}}\left(\left\|A_{Q} \vec{s}_{Q}\right\|_{\mathcal{H}}\right) \chi_{Q}\right\|_{L^{p}(d t)}\right\}_{\nu}\right\|_{l^{q}}<\infty .
$$

Theorem 1.9. Let $\alpha \in \mathbb{R}, 0<q \leq \infty, 1 \leq p<\infty$. Suppose $W$ satisfies any of (A1)-(A3). Then

$$
\|\vec{f}\|_{\dot{B}_{p}^{\alpha q}(W)} \approx\left\|\left\{\vec{s}_{Q}(\vec{f})\right\}_{Q}\right\|_{\dot{b}_{p}^{\alpha q}\left(\left\{A_{Q}\right\}\right)} .
$$

Next we study operators on $\dot{B}_{p}^{\alpha q}(W)$ by considering corresponding operators on $\dot{b}_{p}^{\alpha q}(W)$. In [3] it was shown that almost diagonal operators are bounded on $\dot{b}_{p}^{\alpha q}$ and, thus, on $\dot{B}_{p}^{\alpha q}$. In Section 8 we define a class of almost diagonal matrices $\mathbf{a d}_{p}^{\alpha q}(\beta)$ for the weighted case and show the boundedness of these matrices on $\dot{b}_{p}^{\alpha q}(W)$ if $W$ is a doubling matrix weight:

Theorem 1.10. Let $\alpha \in \mathbb{R}, 0<q \leq \infty, 1 \leq p<\infty$, and let $W$ be a doubling matrix of order $p$ with doubling exponent $\beta$. Consider $A \in \operatorname{ad}_{p}^{\alpha q}(\beta)$. Then $A$ : $\dot{b}_{p}^{\alpha q}(W) \longrightarrow \dot{b}_{p}^{\alpha q}(W)$ is bounded.

We say that a linear continuous operator $T: \mathcal{S} \rightarrow \mathcal{S}^{\prime}$ is almost diagonal, $T \in$ $\mathbf{A D}_{p}^{\alpha q}(\beta)$, if for some pair of mutually admissible kernels $(\varphi, \psi)$ (see (2.1), Section 2) the matrix $\left(\left\langle T \psi_{P}, \varphi_{Q}\right\rangle_{Q P}\right)_{Q, P \text { dyadic }} \in \operatorname{ad}_{p}^{\alpha q}(\beta)$ (see Section [8). Combining the boundedness of an almost diagonal matrix with the norm equivalence, we obtain the boundedness of an almost diagonal operator on $\dot{B}_{p}^{\alpha q}(W)$ under any of (A1)-(A3):

Corollary 1.11. Let $T \in \mathbf{A D}_{p}^{\alpha q}(\beta), \alpha \in \mathbb{R}, 0<q<\infty, 1 \leq p<\infty$. Then $T$ is a bounded operator on $\dot{B}_{p}^{\alpha q}(W)$ if $W$ satisfies any of (A1)-(A3). 
In Section 9 we consider classical convolution and generalized non-convolution Calderón-Zygmund operators (CZOs). The following criterion is used: if an operator $T$ maps "smooth atoms" into "smooth molecules" (see Sections 5 and 9 for definitions), then $T$ is almost diagonal (Lemma 9.2) and, therefore, bounded on $\dot{B}_{p}^{\alpha q}(W)$. To show this property for a CZO, the definition of a "smooth molecule" is modified in order to compensate for the growth of the weight $W$ (note the dependence of the decay rate of the molecule on the doubling exponent $\beta$ ), and, thus, more smoothness of a CZO kernel is required (see Theorems 9.14, 9.8). In particular, for example, we obtain the boundedness of the Hilbert transform (when the underlying dimension is $n=1)$ and the Riesz transforms $(n \geq 2)$ on $\dot{B}_{p}^{\alpha q}(W)$ under any of the conditions (A1)-(A3).

In Section 10 we apply the previous results to Meyer's wavelets and Daubechies' $D N$ wavelets with $N$ sufficiently large, to show that, instead of the $\varphi$-transform coefficients, one can use the wavelet coefficients for the norm equivalence:

Theorem 1.12. If $W$ satisfies any of (A1)-(A3), $\alpha \in \mathbb{R}, 0<q \leq \infty, 1 \leq p<\infty$, then

$$
\|\vec{f}\|_{\dot{B}_{p}^{\alpha q}(W)} \approx\left\|\left\{\vec{s}_{Q}(\vec{f})\right\}_{Q}\right\|_{\dot{b}_{p}^{\alpha q}(W)}
$$

where $\left\{\vec{s}_{Q}(\vec{f})\right\}_{Q}$ are the wavelet coefficients of $\vec{f}$.

So far we have dealt only with homogeneous spaces. However, for a number of applications it is necessary to consider the inhomogeneous distribution spaces (e.g., localized Hardy spaces $H_{\mathrm{loc}}^{p}=F_{p}^{02}, 0<p<\infty$, in particular, $H_{\mathrm{loc}}^{2}=B_{2}^{02}$, [7]). In the last section, we "transfer" the theory developed up until now to the inhomogeneous Besov spaces. The main difference is that instead of considering all dyadic cubes, we consider only the ones with side length $l(Q) \leq 1$, and the properties of functions corresponding to $l(Q)=1$ are slightly changed. Modifying the definitions of the $\varphi$-transform and smooth molecules, we show that all the statements from the homogeneous case are essentially the same for the inhomogeneous spaces.

\section{Notation AND DEFinitions}

Let $z \in \mathbb{R}^{n}$. Recall that $B(z, \delta)=\left\{x \in \mathbb{R}^{n}:|z-x|<\delta\right\} \equiv B_{\delta}(z)$. If the center $z$ of the ball is not essential, we will write $B_{\delta}$ for simplicity.

For each admissible $\varphi \in \mathcal{A}$, there exists $\psi \in \mathcal{A}$ (see e.g. [3]) such that

$$
\sum_{\nu \in \mathbb{Z}} \overline{\hat{\varphi}\left(2^{\nu} \xi\right)} \cdot \hat{\psi}\left(2^{\nu} \xi\right)=1, \text { if } \xi \neq 0
$$

A pair $(\varphi, \psi)$ with $\varphi, \psi \in \mathcal{A}$ and the property (2.1) will be referred to as a pair of mutually admissible kernels.

Similarly to $\varphi_{Q}$, define $\psi_{Q}(x)=|Q|^{-1 / 2} \psi\left(2^{\nu} x-k\right)$ for $Q=Q_{\nu k}$. The inverse $\varphi$-transform $T_{\psi}$ is the map taking a sequence $s=\left\{s_{Q}\right\}_{Q}$ to $T_{\psi} s=\sum_{Q} s_{Q} \psi_{Q}$. In the vector case, $T_{\psi} \vec{s}=\sum_{Q} \vec{s}_{Q} \psi_{Q}$, where $\vec{s}_{Q} \psi_{Q}=\left(s_{Q}^{(1)} \psi_{Q}, \ldots, s_{Q}^{(m)} \psi_{Q}\right)^{\mathrm{T}}$. The $\varphi$-transform decomposition (see [5] for more details) states that for all $f \in \mathcal{S}^{\prime} / \mathcal{P}$,

$$
f=\sum_{Q}\left\langle f, \varphi_{Q}\right\rangle \psi_{Q}=: \sum_{Q} s_{Q} \psi_{Q}
$$


In other words, $T_{\psi} \circ S_{\varphi}$ is the identity on $\mathcal{S}^{\prime} / \mathcal{P}$. Observe that if $\tilde{\varphi}(x)=\overline{\varphi(-x)}$ (note that $\tilde{\varphi} \in \mathcal{A})$, then $s_{Q}=\left\langle f, \varphi_{Q}\right\rangle=|Q|^{1 / 2}\left(\tilde{\varphi}_{\nu} * f\right)\left(2^{-\nu} k\right)$.

\section{Matrix $A_{p}$ CONDition}

Although the $A_{p}$ condition can be formulated for any family of norms $\rho_{t}$ on a Hilbert space, we will consider only the particular case of norms:

$$
\rho_{t}(x)=\left\|W^{1 / p}(t) x\right\|, \quad \text { where } x \in \mathcal{H}, t \in \mathbb{R}^{n} .
$$

Then the dual metric $\rho^{*}$ is given by

$$
\rho_{t}^{*}(x)=\sup _{y \neq 0} \frac{|(x, y)|}{\rho_{t}(y)}=\left\|W^{-1 / p}(t) x\right\| .
$$

Following [17, we introduce the norms $\rho_{p, B}$ through the averagings of the metrics $\rho_{t}$ over a ball $B$ :

$$
\rho_{p, B}(x)=\left(\frac{1}{|B|} \int_{B}\left[\rho_{t}(x)\right]^{p} d t\right)^{1 / p} .
$$

Similarly, for the dual metric,

$$
\rho_{p^{\prime}, B}^{*}(x)=\left(\frac{1}{|B|} \int_{B}\left[\rho_{t}^{*}(x)\right]^{p^{\prime}} d t\right)^{1 / p^{\prime}} .
$$

Definition 3.1 (Matrix $A_{p}$ weight). For $1<p<\infty$, we say that $W$ is an $A_{p}$ matrix weight if $W: \mathbb{R}^{n} \rightarrow \mathcal{M}$ is such that $W$ and $W^{-p^{\prime} / p}$ are locally integrable and there exists $C<\infty$ such that

$$
\rho_{p^{\prime}, B}^{*} \leq C\left(\rho_{p, B}\right)^{*} \quad \text { for every ball } B \subseteq \mathbb{R}^{n} .
$$

In general, if $\rho$ satisfies (3.1), then $\rho$ is called an $A_{p}$-metric. Note that the condition (3.1) is equivalent to

$$
\rho_{p, B} \leq C\left(\rho_{p^{\prime}, B}^{*}\right)^{*},
$$

which means that $\rho^{*}$ is an $A_{p^{\prime}}$-metric.

If $\rho$ is a norm on $\mathcal{H}$, then there exists a positive operator $A$, which is called a reducing operator of $\rho$, such that

$$
\rho(x) \approx\|A x\| \quad \text { for all } x \in \mathcal{H} .
$$

For details we refer the reader to [17]. Let $A_{B}$ be a reducing operator for $\rho_{p, B}$, and $A_{B}^{\#}$ for $\rho_{p^{\prime}, B}^{*}$. Then, $\left(\rho_{p, B}\right)^{*}(x) \approx\left\|A_{B}^{-1} x\right\|$. Hence, in the language of reducing operators, the condition (3.1) for the $A_{p}$ class is

$$
\left\|A_{B}^{\#} A_{B}\right\| \leq C<\infty \text { for every ball } B \subseteq \mathbb{R}^{n} .
$$

Observe the following two useful facts. First, if $P$ and $Q$ are two selfadjoint operators in a normed space, then

$$
\|P Q\|=\left\|(P Q)^{*}\right\|=\left\|Q^{*} P^{*}\right\|=\|Q P\| .
$$

Thus, the operators can be commuted as long as we deal with norms.

Second, we need the following lemma: 
Lemma 3.2 (NORM LEMMA). If $\left\{e_{1}, \ldots, e_{m}\right\}$ is any orthonormal basis in a Hilbert space $\mathcal{H}$, then for any $V \in \mathcal{B}(\mathcal{H})$ and $r>0$,

$$
\|V\|^{r} \underset{(r, m)}{\approx} \sum_{i=1}^{m}\left\|V e_{i}\right\|_{\mathcal{H}}^{r} .
$$

Proof. With $x_{i}=\left(x, e_{i}\right)_{\mathcal{H}}$, we get

$$
\begin{aligned}
\|V\|^{r} & =\sup _{\|x\| \leq 1}\left\|V \sum_{i=1}^{m} x_{i} e_{i}\right\|_{\mathcal{H}}^{r} \\
& \leq c_{r} \sup _{\|x\| \leq 1} \sum_{i=1}^{m}\left|x_{i}\right|^{r}\left\|V e_{i}\right\|_{\mathcal{H}}^{r} \leq c_{r} \sum_{i=1}^{m}\left\|V e_{i}\right\|_{\mathcal{H}}^{r} \leq c_{r} m\|V\|^{r} .
\end{aligned}
$$

Now we are ready to prove the equivalence of $(1.2)$ and the $A_{p}$ condition.

Proof of Lemma 1.3. By property (3.3) and the Norm Lemma,

$$
\begin{aligned}
\int_{B} & \left(\int_{B}\left\|W^{1 / p}(x) W^{-1 / p}(t)\right\|^{p^{\prime}} \frac{d t}{|B|}\right)^{p / p^{\prime}} \frac{d x}{|B|} \\
& =\int_{B}\left(\int_{B}\left\|W^{-1 / p}(t) W^{1 / p}(x)\right\|^{p^{\prime}} \frac{d t}{|B|}\right)^{p / p^{\prime}} \frac{d x}{|B|} \\
& \approx \int_{B}\left(\int_{B} \sum_{i=1}^{m}\left\|W^{-1 / p}(t) W^{1 / p}(x) e_{i}\right\|^{p^{\prime}} \frac{d t}{|B|}\right)^{p / p^{\prime}} \frac{d x}{|B|} \\
& \approx \sum_{i=1}^{m} \int_{B}\left(\int_{B}\left[\rho_{t}^{*}\left(W^{1 / p}(x) e_{i}\right)\right]^{p^{\prime}} \frac{d t}{|B|}\right)^{p / p^{\prime}} \frac{d x}{|B|} \\
& =\sum_{i=1}^{m} \int_{B}\left[\rho_{p^{\prime}, B}^{*}\left(W^{1 / p}(x) e_{i}\right)\right]^{p} \frac{d x}{|B|} .
\end{aligned}
$$

Now, in terms of the reducing operators, the last expression is equivalent to

$$
\begin{aligned}
\sum_{i=1}^{m} \int_{B}\left\|A_{B}^{\#}\left(W^{1 / p}(x) e_{i}\right)\right\|^{p} \frac{d x}{|B|} & \approx \int_{B}\left\|A_{B}^{\#} W^{1 / p}(x)\right\|^{p} \frac{d x}{|B|} \\
& \approx \sum_{i=1}^{m} \int_{B}\left\|W^{1 / p}(x)\left(A_{B}^{\#} e_{i}\right)\right\|^{p} \frac{d x}{|B|} \\
& \approx \sum_{i=1}^{m}\left[\rho_{p, B}\left(A_{B}^{\#} e_{i}\right)\right]^{p} \\
& \approx \sum_{i=1}^{m}\left\|A_{B}\left(A_{B}^{\#} e_{i}\right)\right\|^{p} \\
& \approx\left\|A_{B} A_{B}^{\#}\right\|^{p}
\end{aligned}
$$

Therefore, (1.2) is equivalent to $\left\|A_{B}^{\#} A_{B}\right\|^{p} \leq c$, i.e., the $A_{p}$ condition.

Corollary 3.3 (Symmetry of MAtrix $A_{p}$ COndition). The following statements are equivalent: 
(i) $W \in A_{p}$;

(ii) $W^{-p^{\prime} / p} \in A_{p^{\prime}}$;

(iii) $\int_{B}\left(\int_{B}\left\|W^{1 / p}(x) W^{-1 / p}(t)\right\|^{p^{\prime}} \frac{d t}{|B|}\right)^{p / p^{\prime}} \frac{d x}{|B|} \leq c \quad$ for every ball $B \subseteq \mathbb{R}^{n}$;

(iv) $\int_{B}\left(\int_{B}\left\|W^{1 / p}(x) W^{-1 / p}(t)\right\|^{p} \frac{d x}{|B|}\right)^{p^{\prime} / p} \frac{d t}{|B|} \leq c \quad$ for every ball $B \subseteq \mathbb{R}^{n}$.

Proof. Recall that $\rho \in A_{p}$ if and only if $\rho^{*} \in A_{p^{\prime}}$. In terms of matrix weights, $W \in A_{p}$ if and only if $W^{-p^{\prime} / p} \in A_{p^{\prime}}$ (note that $\rho_{t}^{*}(x)=\left\|\left(W^{-p^{\prime} / p}\right)^{1 / p^{\prime}}(t) x\right\|$ ). By Lemma 1.3 the third statement is equivalent to $W \in A_{p}$, whereas the fourth is equivalent to $W^{-p^{\prime} / p} \in A_{p^{\prime}}$.

\section{Doubling Measures}

Let $W$ be a doubling matrix of order $p$, i.e., (1.5) holds for any $y \in \mathcal{H}, \delta>0$ and $z \in \mathbb{R}^{n}$. For $p=2$ this simplifies to

$$
\int_{B_{2 \delta}} W(t) d t \leq c \int_{B_{\delta}} W(t) d t
$$

for a given $\delta$, where the inequality is understood in the sense of selfadjoint operators.

Remark 4.1. Note that $\left\|W^{1 / p}(t)\right\|^{p}$ is independent of $p$. If $w_{y}(t)=\left\|W^{1 / p}(t) y\right\|_{\mathcal{H}}^{p}$ is doubling of order $p$ for any $y \in \mathcal{H}$, then $w(t)=\left\|W^{1 / p}(t)\right\|^{p}$ is also a scalar-valued doubling measure.

Proof. Fix $t \in \mathbb{R}^{n}$. Then there exist a unitary matrix $U$ and a diagonal matrix $\Lambda$ such that $W(t)=U \Lambda U^{-1}$, and so $W^{1 / p}(t)=U \Lambda^{1 / p} U^{-1}$. Moreover, since the norm of a positive diagonal matrix is the largest eigenvalue, say $\lambda_{0},\left\|W^{1 / p}(t)\right\|=$ $\lambda_{0}^{1 / p}$ and, hence, $\left\|W^{1 / p}(t)\right\|^{p}=\lambda_{0}$, regardless of what $p$ is.

Now, since (1.5) is true with $y=e_{i}$ (any orthonormal basis vector of $\mathcal{H}$ ), by the Norm Lemma we get the second assertion:

$$
\begin{gathered}
\int_{B_{2 \delta}}\left\|W^{1 / p}(t)\right\|^{p} d t \approx \sum_{i=1}^{m} \int_{B_{2 \delta}}\left\|W^{1 / p}(t) e_{i}\right\|^{p} d t \\
\leq c \sum_{i=1}^{m} \int_{B_{\delta}}\left\|W^{1 / p}(t) e_{i}\right\|^{p} d t \approx c \int_{B_{\delta}}\left\|W^{1 / p}(t)\right\|^{p} d t .
\end{gathered}
$$

The doubling property of $w(t)=\left\|W^{1 / p}(t)\right\|^{p}$ is not very helpful if one wants to understand the nature of $W$; it only tells us how large the weight is, not how it is distributed in different directions. Therefore, we use the definition of doubling matrix in (1.5), which involves different directions of $y \in \mathcal{H}$.

Remark 4.2. In the scalar case, (1.5) gives the standard doubling measure:

$$
\int_{B_{2 \delta}} w(t)|y|^{p} d t \leq c \int_{B_{\delta}} w(t)|y|^{p} d t
$$

and if $y \neq 0$, then $w\left(B_{2 \delta}\right) \leq c w\left(B_{\delta}\right)$. In particular, there is no dependence on $p$ in the scalar situation. 
Similar definitions for doubling weights can be analogously given for the "dual" measure $w_{y}^{*}(t)=\left\|W^{-1 / p}(t) y\right\|^{p^{\prime}}$.

Lemma 4.3. Let $x \in \mathcal{H}$ and $W \in A_{p}$. Then $v_{x}(t):=\left\|W^{1 / p}(x) W^{-1 / p}(t)\right\|^{p^{\prime}}=$ $\left\|W^{-1 / p}(t) W^{1 / p}(x)\right\|^{p^{\prime}}$ is a doubling measure, i.e., there exists a constant $c$ such that for any $\delta>0$,

$$
\int_{B_{2 \delta}}\left\|W^{1 / p}(x) W^{-1 / p}(t)\right\|^{p^{\prime}} d t \leq c \int_{B_{\delta}}\left\|W^{1 / p}(x) W^{-1 / p}(t)\right\|^{p^{\prime}} d t .
$$

Proof. Applying the Norm Lemma to the operator norm in the left-hand side, we obtain

$$
v_{x}(t) \approx \sum_{i=1}^{m}\left\|W^{-1 / p}(t) W^{1 / p}(x) e_{i}\right\|^{p^{\prime}}=\sum_{i=1}^{m}\left\|W^{-1 / p}(t) y_{i}(x)\right\|^{p^{\prime}}=\sum_{i=1}^{m} w_{y_{i}(x)}^{*}(t),
$$

where $y_{i}(x)=W^{1 / p}(x) e_{i}$. Then

$$
v_{x}\left(B_{2 \delta}\right) \approx \sum_{i=1}^{m} \int_{B_{2 \delta}} w_{y_{i}(x)}^{*}(t) d t \leq \sum_{i=1}^{m} c \int_{B_{\delta}} w_{y_{i}(x)}^{*}(t) d t \leq c v_{x}\left(B_{\delta}\right)
$$

since $w_{y}^{*}$ is doubling $\left(W^{-p^{\prime} / p} \in A_{p^{\prime}}\right)$.

Remark 4.4. The doubling property (1.4) is equivalent to

$$
\frac{\mu(F)}{\mu(E)} \leq c\left(\frac{|F|}{|E|}\right)^{\beta / n}
$$

where $F$ is a ball (or a cube) and $E \subseteq F$ is a sub-ball (sub-cube) (not any subset of $F$; any subset would be equivalent to the $A_{\infty}$ condition, see [14]).

Proof. Since $E \subseteq F$, there exists $j \in \mathbb{N}$ such that $2^{j} E \approx F$, i.e., $l(F) \approx 2^{j} l(E)$.

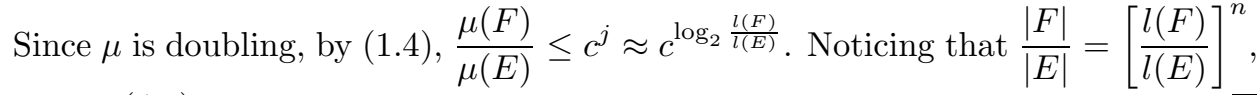
we get (4.3).

In further estimates, it is more convenient to use (4.3) instead of (1.4).

Observe that the doubling exponent of the Lebesgue measure in $\mathbb{R}^{n}$ is $\beta=n$; moreover, if $\mu$ is any nonzero doubling measure in $\mathbb{R}^{n}$, then $\beta(\mu) \geq n$.

\section{BOUNDEDNESS OF THE INVERSE $\varphi$-TRANSFORM}

Consider $\dot{B}_{p}^{\alpha q}(W)$ with parameters $\alpha, p, q$ fixed $(\alpha \in \mathbb{R}, 1 \leq p<\infty, 0<q<\infty)$. For $0<\delta \leq 1, M>0$ and $N \in \mathbb{Z}$ define (as in [5]) $m_{Q}$ to be a smooth $(\delta, M, N)$ )molecule for $Q$ dyadic if:

$$
\begin{aligned}
& \text { (M1) } \int x^{\gamma} m_{Q}(x) d x=0, \text { for }|\gamma| \leq N, \\
& \text { (M2) }\left|m_{Q}(x)\right| \leq|Q|^{-1 / 2}\left(1+\frac{\left|x-x_{Q}\right|}{l(Q)}\right)^{-\max (M, M-\alpha)}, \\
& \text { (M3) }\left|D^{\gamma} m_{Q}(x)\right| \leq|Q|^{-1 / 2-|\gamma| / n}\left(1+\frac{\left|x-x_{Q}\right|}{l(Q)}\right)^{-M} \text { if }|\gamma| \leq[\alpha], \\
& \text { (M4) }\left|D^{\gamma} m_{Q}(x)-D^{\gamma} m_{Q}(y)\right| \leq|Q|^{-\frac{1}{2}-\frac{|\gamma|}{n}-\frac{\delta}{n}}|x-y|^{\delta} \\
& \quad \times \sup _{|z| \leq|x-y|}\left(1+\frac{\left|x-z-x_{Q}\right|}{l(Q)}\right)^{-M} \text { if }|\gamma|=[\alpha] .
\end{aligned}
$$


It is understood that (M1) is void if $N<0$; and (M3), (M4) are void if $\alpha<0$. Also, $[\alpha]$ stands for the greatest integer $\leq \alpha ; \gamma$ is a multi-index $\gamma=\left(\gamma_{1}, \ldots, \gamma_{n}\right)$ with $\gamma_{i} \in \mathbb{N} \cup\{0\}, 1 \leq i \leq n$, and the standard notation is used.

We say $\left\{m_{Q}\right\}_{Q}$ is a family of smooth molecules for $\dot{B}_{p}^{\alpha q}(W)$ if each $m_{Q}$ is a $(\delta, M, N)$-molecule with

(M.i) $\alpha-[\alpha]<\delta \leq 1$,

(M.ii) $M>J$, where $J=\frac{\beta}{p}+\frac{n}{p^{\prime}}$ if $p>1$ and $J=\beta$ if $p=1$,

(M.iii) $N=\max ([J-n-\alpha],-1)$.

Remark 5.1. Note that, in contrast to the case in [5], there is a dependence of the family of smooth molecules for $\dot{B}_{p}^{\alpha q}(W)$ on the weight $W$ (more precisely, on the doubling exponent $\beta$ ).

Theorem 5.2. Let $\alpha \in \mathbb{R}, 1 \leq p<\infty, 0<q \leq \infty$, and let $W$ be a doubling matrix weight of order $p$. Suppose $\left\{m_{Q}\right\}_{Q}$ is a family of smooth molecules for $\dot{B}_{p}^{\alpha q}(W)$. Then

$$
\left\|\sum_{Q} \vec{s}_{Q} m_{Q}\right\|_{\dot{B}_{p}^{\alpha q}(W)} \leq c\left\|\left\{\vec{s}_{Q}\right\}_{Q}\right\|_{\dot{b}_{p}^{\alpha q}(W)} .
$$

The proof uses the following estimates for $Q$ dyadic with $l(Q)=2^{-\mu}, \mu \in \mathbb{Z}$, and $\varphi_{\nu}, \nu \in \mathbb{Z}$, with $\varphi \in \mathcal{A}$ :

if $\mu>\nu$, and

$$
\left|\varphi_{\nu} * m_{Q}(x)\right| \leq c|Q|^{-1 / 2} 2^{-(\nu-\mu) \tau}\left(1+2^{\mu}\left|x-x_{Q}\right|\right)^{-M} \text { for some } \tau>\alpha,
$$

if $\mu \leq \nu$.

The proofs are entirely elementary, but quite tedious (see [5], Appendix B). Note that in the statement of Lemma B.1 in $[5$, it should say $j \leq k$. For (5.2), for $N \neq-1$, apply Lemma B.1 with $j=\nu, k=\mu, L=N, R=M, S=M-\alpha$, $g=2^{-\nu n / 2} \varphi_{\nu}, h=m_{Q}$ with $l(Q)=2^{-\mu}, x_{1}=x_{Q}, J-n-\alpha-[J-n-\alpha]<\theta \leq 1$. Letting $\sigma=N+n+\theta>J-\alpha$, we obtain (5.2). For $N=-1$, apply Lemma B.2 in [5] with $\sigma=n>J-\alpha$ to get (5.2). Now for (5.3), for $\alpha>0$, apply Lemma B.1 with $k=\nu, j=\mu, L=[\alpha], R=M, \delta=\theta, S=[\alpha]+n+\delta, x_{1}=0, g(x)=m_{Q}\left(x+x_{Q}\right)$, $h=2^{-\nu n / 2} \varphi_{\nu}$, and observe that $\varphi_{\nu} * m_{Q}(x)=2^{\nu n / 2} g * h\left(x-x_{Q}\right)$ to get (5.3) with $\tau=\delta+[\alpha]>\alpha$. For $\alpha<0$, Lemma B.2 in [5] gives (5.3) with $\tau=0>\alpha$.

Lemma 5.3 (Squeeze Lemma). Fix a dyadic cube $Q$ and let $w: \mathbb{R}^{n} \rightarrow \mathbb{R}^{+}$be a scalar doubling measure with the doubling exponent $\beta$. If $L>\beta$, then for $r \geq l(Q)$,

$$
\int_{\mathbb{R}^{n}} w(x)\left(1+\frac{\left|x-x_{Q}\right|}{r}\right)^{-L} d x \leq c_{\beta}\left[\frac{r}{l(Q)}\right]^{\beta} \int_{Q} w(x) d x .
$$

Proof. Decompose $\mathbb{R}^{n}$ into the annuli $\mathcal{R}_{m}$ :

$$
\mathbb{R}^{n}=\bigcup_{m=1}^{\infty}\left\{x: 2^{m-1} r \leq\left|x-x_{Q}\right|<2^{m} r\right\} \cup\left\{x:\left|x-x_{Q}\right|<r\right\}=: \bigcup_{m=0}^{\infty} \mathcal{R}_{m} .
$$

Then the left-hand side of (5.4) is bounded by

$$
\sum_{m=1}^{\infty}\left(1+2^{m-1}\right)^{-L} w\left(\mathcal{R}_{m}\right)+w\left(\mathcal{R}_{0}\right) .
$$


Using the doubling property of $w$, we get

$$
w\left(\mathcal{R}_{m}\right) \leq w\left(B\left(x_{Q}, 2^{m} r\right)\right) \leq c\left(\frac{\left|B\left(x_{Q}, 2^{m} r\right)\right|}{\left|\mathcal{R}_{0}\right|}\right)^{\beta / n} w\left(\mathcal{R}_{0}\right)=c 2^{m \beta} w\left(\mathcal{R}_{0}\right) .
$$

Thus, (5.5) is bounded by

$$
c \sum_{m=0}^{\infty} 2^{m \beta-m L} w\left(\mathcal{R}_{0}\right) \leq c_{\beta} w\left(\mathcal{R}_{0}\right),
$$

since $L>\beta$. Note that $B\left(x_{Q}, l(Q)\right) \subseteq 3 Q$ and so $w\left(B\left(x_{Q}, l(Q)\right)\right) \leq c_{\beta} w(Q)$. If $r>l(Q)$, then

$$
w\left(\mathcal{R}_{0}\right) \leq c\left(\frac{\left|\mathcal{R}_{0}\right|}{\left|B\left(x_{Q}, l(Q)\right)\right|}\right)^{\beta / n} w\left(B\left(x_{Q}, l(Q)\right)\right) \leq c_{\beta}\left[\frac{r}{l(Q)}\right]^{\beta} w(Q),
$$

which is (5.4).

Lemma 5.4 (Summation Lemma). Let $\mu, \nu \in \mathbb{Z}$ and $y \in \mathbb{R}^{n}$. Then for $M>n$,

$$
\sum_{l(Q)=2^{-\mu}}\left(1+\frac{\left|y-x_{Q}\right|}{2^{-\nu}}\right)^{-M} \leq c_{n, M} 2^{(\mu-\nu) n}, \text { if } \mu \geq \nu .
$$

Proof. If $\mu \geq \nu$ or $2^{-\nu} \geq 2^{-\mu}$, there are $2^{(\mu-\nu) n}$ cubes of size $2^{-\mu}$ in a cube of size $2^{-\nu}$. Fix $l \in \mathbb{Z}^{n}$ such that $y \in Q_{\nu l}$. Then the left-hand side of (5.6) is

$$
\begin{aligned}
\sum_{k \in \mathbb{Z}^{n}}\left(1+2^{\nu}\left|y-x_{Q_{\mu k}}\right|\right)^{-M} & =\sum_{i \in \mathbb{Z}^{n}} \sum_{k: Q_{\mu k} \subseteq Q_{\nu(l+i)}}\left(1+2^{\nu}\left|y-x_{Q_{\mu k}}\right|\right)^{-M} \\
& \leq \sum_{i \in \mathbb{Z}^{n}}(1+|i|)^{-M} \times 2^{(\mu-\nu) n} \leq c_{n} 2^{(\mu-\nu) n}
\end{aligned}
$$

again since $M>n$.

Proof of Theorem 5.2. By definition,

$$
\begin{aligned}
\left\|\sum_{Q} \vec{s}_{Q} m_{Q}\right\|_{\dot{B}_{p}^{\alpha q}(W)} & \left.\left.=\left\|\left\{\left\|W^{1 / p} \sum_{Q} \vec{s}_{Q}\left(\varphi_{\nu} * m_{Q}\right)\right\|_{L^{p}}\right\}_{\nu}\right\|_{l_{q}^{\alpha}}\right]\|\|_{L^{p}}\right\}_{\nu} \|_{l_{q}^{\alpha}} . \\
& =\|\left\{\sum_{\mu \in \mathbb{Z}}\left[\sum_{l(Q)=2^{-\mu}}\left(W^{1 / p} \vec{s}_{Q}\right)\left(\varphi_{\nu} * m_{Q}\right)\right]\right.
\end{aligned}
$$

By Minkowski's (or the triangle) inequality, the last expression is bounded by

$$
\begin{gathered}
\left\|\left\{\sum_{\mu \in \mathbb{Z}}\left\|\sum_{l(Q)=2^{-\mu}}\left(W^{1 / p} \vec{s}_{Q}\right)\left(\varphi_{\nu} * m_{Q}\right)\right\|_{L^{p}}\right\}_{\nu}\right\|_{l_{q}^{\alpha}} \|_{\nu} \\
\leq \|\left\{\sum_{\mu \in \mathbb{Z}}\left(\int_{\mathbb{R}^{n}}\left(\sum_{l(Q)=2^{-\mu}}\left\|W^{1 / p}(x) \vec{s}_{Q}\right\|\left|\left(\varphi_{\nu} * m_{Q}\right)(x)\right|\right)^{1 / p} d x\right)_{\nu} \|_{l_{q}^{\alpha}}\right.
\end{gathered}
$$




$$
=:\left\|\left\{\sum_{\mu>\nu} J_{1}^{1 / p}+\sum_{\mu \leq \nu} J_{2}^{1 / p}\right\}_{\nu}\right\|_{l_{q}^{\alpha}} .
$$

Using estimates (5.2) and (5.3) with $\theta_{1}=-(\mu-\nu) \sigma, \theta_{2}=-(\nu-\mu) \tau$ and $r_{1}=2^{-\nu}$, $r_{2}=2^{-\mu}$, we bound each $J_{i}, i=1,2$ :

$$
J_{i} \leq c \int_{\mathbb{R}^{n}}\left(\sum_{l(Q)=2^{-\mu}}\left\|W^{1 / p}(x) \vec{s}_{Q}\right\|_{\mathcal{H}}|Q|^{-1 / 2} 2^{\theta_{i}}\left(1+\frac{\left|x-x_{Q}\right|}{r_{i}}\right)^{-M}\right)^{p} d x .
$$

Split $M=M_{1}+M_{2}$, where $M_{1}>\beta / p$ and $M_{2}>n / p^{\prime}$ (if $p=1$, let $M_{2}=0$ and interpret $\left.n / p^{\prime}=0\right)$. This splitting is possible since $M>J$. Then by the discrete Hölder inequality with $w_{Q}(x)=\left\|W^{1 / p}(x) \vec{s}_{Q}\right\|_{\mathcal{H}}^{p}$, we get

$$
\begin{aligned}
J_{i} & \leq c_{p} \int_{\mathbb{R}^{n}}\left(\sum_{l(Q)=2^{-\mu}} w_{Q}(x)|Q|^{-p / 2} 2^{\theta_{i} p}\left(1+\frac{\left|x-x_{Q}\right|}{r_{i}}\right)^{-M_{1} p}\right) \\
& \times\left[\sum_{l(Q)=2^{-\mu}}\left(1+\frac{\left|x-x_{Q}\right|}{r_{i}}\right)^{-M_{2} p^{\prime}}\right]^{p / p^{\prime}} d x .
\end{aligned}
$$

By the Summation Lemma 5.4 (with $\nu=\mu$ in (5.6)), we have

$$
J_{2} \leq c_{p, n} 2^{\theta_{2} p} \sum_{l(Q)=2^{-\mu}}|Q|^{-p / 2} \int_{\mathbb{R}^{n}} w_{Q}(x)\left(1+2^{\mu}\left|x-x_{Q}\right|\right)^{-M_{1} p} d x,
$$

since $M_{2}>n / p^{\prime}$. Applying the Squeeze Lemma 5.3 with $r=2^{-\mu}=l(Q)$ and $L=M_{1} p$ (and so $L>\beta$ ), we get

$$
J_{2} \leq c_{p, n, \beta} 2^{-(\nu-\mu) \tau p} \sum_{l(Q)=2^{-\mu}}|Q|^{-p / 2} w_{Q}(Q) .
$$

By the Summation Lemma 5.4 (with $\mu>\nu$ in (5.6)), we have

$$
J_{1} \leq c_{p, n} 2^{(\nu-\mu)\left(\sigma-n / p^{\prime}\right) p} \sum_{l(Q)=2^{-\mu}}|Q|^{-p / 2} \int_{\mathbb{R}^{n}} w_{Q}(x)\left(1+2^{\nu}\left|x-x_{Q}\right|\right)^{-M_{1} p} d x,
$$

again since $M_{2}>n / p^{\prime}$. Applying the Squeeze Lemma 5.3 again with $r=2^{-\nu}>$ $2^{-\mu}=l(Q)$ and $L=M_{1} p$, we get

$$
J_{1} \leq c_{p, n, \beta} 2^{(\nu-\mu)\left(\sigma-n / p^{\prime}-\beta / p\right) p} \sum_{l(Q)=2^{-\mu}}|Q|^{-p / 2} w_{Q}(Q) .
$$

Observe that the last sum is equal to $\left\|\sum_{l(Q)=2^{-\mu}}|Q|^{-1 / 2} \vec{s}_{Q} \chi_{Q}\right\|_{L^{p}(W)}^{p}$. Combining the estimates for $J_{1}$ and $J_{2}$ (recall that $J=\frac{n}{p^{\prime}}+\frac{\beta}{p}$ ), we have

$$
\begin{gathered}
2^{\nu \alpha}\left(\sum_{\mu>\nu} J_{1}^{1 / p}+\sum_{\mu \leq \nu} J_{2}^{1 / p}\right) \leq c_{p, n, \beta} \sum_{\mu \in \mathbb{Z}} 2^{(\nu-\mu) \alpha}\left(2^{(\nu-\mu)(\sigma-J)} \chi_{\{\nu-\mu<0\}}\right. \\
\left.+2^{-(\nu-\mu) \tau} \chi_{\{\nu-\mu \geq 0\}}\right) \times 2^{\mu \alpha}\left\|\sum_{l(Q)=2^{-\mu}}|Q|^{-1 / 2} \vec{s}_{Q} \chi_{Q}\right\|_{L^{p}(W)} .
\end{gathered}
$$


Denote

$$
a_{i}=2^{i \alpha}\left(2^{i(\sigma-J)} \chi_{\{i<0\}}+2^{-i \tau} \chi_{\{i \geq 0\}}\right)
$$

and

$$
b_{\mu}=2^{\mu \alpha}\left\|\sum_{l(Q)=2^{-\mu}}|Q|^{-1 / 2} \overrightarrow{s_{Q}} \chi_{Q}\right\|_{L^{p}(W)} .
$$

Then the right side of (15.8) is nothing else but $c \sum_{\mu \in \mathbb{Z}} a_{\nu-\mu} b_{\mu}=c(a * b)(\nu)$. Substituting this into (5.7), we get

$$
\left\|\sum_{Q} \vec{s}_{Q} m_{Q}\right\|_{\dot{B}_{p}^{\alpha q}(W)} \leq\left\|\left\{\sum_{\mu \in \mathbb{Z}} \sum_{i=1,2} J_{i}^{1 / p}\right\}_{\nu}\right\|_{l_{q}^{\alpha}} \leq c_{p, n, \beta}\|a * b\|_{l^{q}} .
$$

Observe that

$$
\|a * b\|_{l^{q}} \leq\|a\|_{l^{1}}\|b\|_{l^{q}} \text { for } q \geq 1
$$

and

$$
\|a * b\|_{l^{q}} \leq\|a\|_{l^{q}}\|b\|_{l^{q}} \text { for } q<1
$$

(to get the last inequality, apply the $q$-triangle inequality followed by $\|a * b\|_{l^{1}} \leq$ $\left.\|a\|_{l^{1}}\|b\|_{l^{1}}\right)$. For any $0<q<\infty, \quad\|a\|_{l^{q}}^{q}=\sum_{i<0} 2^{i(\sigma+\alpha-J) q}+\sum_{i \geq 0} 2^{-i(\tau-\alpha) q}$. Both sums converge, since $\tau>\alpha$ and $\sigma+\alpha>J$ by (5.2) and (15.3). Hence, $\|a\|_{l^{q}} \leq c_{q}$ for any $q>0$. (In fact, here we only need $0<q \leq 1$.) Combining all the estimates together into (5.9), we obtain

$$
\begin{aligned}
\left\|\sum_{Q} \vec{s}_{Q} m_{Q}\right\|_{\dot{B}_{p}^{\alpha q}(W)} \leq c\|b\|_{l^{q}} & =c\left\|\left\{2^{\nu \alpha}\left\|\sum_{l(Q)=2^{-\nu}}|Q|^{-1 / 2} \vec{s}_{Q} \chi_{Q}\right\|_{L^{p}(W)}\right\}_{\nu}\right\|_{l^{q}} \\
& =c\left\|\left\{\vec{s}_{Q}\right\}\right\|_{\dot{b}_{p}^{\alpha q}(W)},
\end{aligned}
$$

where $c=c_{n, p, q, \beta}$.

Remark 5.5. Since $\psi \in \mathcal{A}$, observe the following properties of $\psi_{Q}$ :

1. $0 \notin \operatorname{supp} \hat{\psi}_{Q}$ for any dyadic $Q$, and, therefore, $\int x^{\gamma} \psi_{Q}(x) d x=0$ for any multi-index $\gamma$;

2. $\left|D^{\gamma} \psi_{Q}\right| \leq c_{\gamma, L}|Q|^{-\frac{1}{2}-\frac{|\gamma|}{n}}\left(1+\frac{\left|x-x_{Q}\right|}{l(Q)}\right)^{-L-|\gamma|}$ for each $L \in \mathbb{N} \cup\{0\}$ and $\gamma$ as before.

Hence, $\left\{\psi_{Q}\right\}_{Q}$ is a family of smooth molecules for $\dot{B}_{p}^{\alpha q}(W)$, and for $\vec{f}=\sum_{Q} \vec{s}_{Q} \psi_{Q}$, we obtain the boundedness of the inverse $\varphi$-transform $T_{\psi}$ :

Corollary 5.6. Let $W$ be a doubling matrix of order $p$, and consider the sequence $\vec{s}=\left\{\vec{s}_{Q}\right\}_{Q} \in \dot{b}_{p}^{\alpha q}(W)$. Then for all $1 \leq p<\infty, 0<q \leq \infty$ and $\alpha \in \mathbb{R}$,

$$
\left\|T_{\psi} \vec{s}\right\|_{\dot{B}_{p}^{\alpha q}(W)}=\left\|\sum_{Q} \vec{s}_{Q} \psi_{Q}\right\|_{\dot{B}_{p}^{\alpha q}(W)} \leq c\left\|\left\{\vec{s}_{Q}\right\}_{Q}\right\|_{\dot{b}_{p}^{\alpha q}(W)} .
$$


In particular, given $\vec{f} \in \dot{B}_{p}^{\alpha q}(W)$, consider $\vec{s}=S_{\varphi} \vec{f}$. Then by (2.2),

$$
\|\vec{f}\|_{\dot{B}_{p}^{\alpha q}(W)}=\left\|\sum_{Q} \vec{s}_{Q} \psi_{Q}\right\|_{\dot{B}_{p}^{\alpha q}(W)} \leq c\left\|\left\{\vec{s}_{Q}\right\}_{Q}\right\|_{\dot{b}_{p}^{\alpha q}(W)}=c\left\|S_{\varphi} \vec{f}\right\|_{\dot{b}_{p}^{\alpha q}(W)} .
$$

\section{BOUNDEDNESS OF THE $\varphi$-TRANSFORM}

Definition 6.1. For $\nu \in \mathbb{Z}$, let $E_{\nu}=\left\{\vec{f}: f_{i} \in \mathcal{S}^{\prime}\right.$ and supp $\hat{f}_{i} \subseteq\left\{\xi \in \mathbb{R}^{n}:|\xi| \leq\right.$ $\left.\left.2^{\nu+1}\right\}, i=1, \ldots, m\right\}$. Then we say that $E_{\nu}$ consists of vector functions of exponential type $2^{\nu+1}$.

Consider the following lemma on the decomposition of an exponential type function (for the proof the reader is referred to [3], p. 55):

Lemma 6.2. Suppose $g \in \mathcal{S}^{\prime}\left(\mathbb{R}^{n}\right), h \in \mathcal{S}\left(\mathbb{R}^{n}\right)$ and $\operatorname{supp} \hat{g}$, supp $\hat{h} \subseteq\left\{|\xi|<2^{\nu} \pi\right\}$ for some $\nu \in \mathbb{Z}$. Then

$$
(g * h)(x)=\sum_{k \in \mathbb{Z}^{n}} 2^{-\nu n} g\left(2^{-\nu} k\right) h\left(x-2^{-\nu} k\right) .
$$

Now we will develop two "maximal operator" type inequalities:

Lemma 6.3. Let $1<p<\infty, W \in A_{p}$ and $\vec{g} \in E_{0}$. Then

$$
\sum_{k \in \mathbb{Z}^{n}} \int_{Q_{0 k}}\left\|W^{1 / p}(x) \vec{g}(k)\right\|^{p} d x \leq c_{p, n}\|\vec{g}\|_{L^{p}(W)}^{p} .
$$

Remark 6.4. Note that in terms of reducing operators, (6.2) is equivalent to

$$
\left\|\left\{A_{Q_{0 k}} \vec{g}(k)\right\}_{k \in \mathbb{Z}^{n}}\right\|_{l^{p}}=\left(\sum_{k \in \mathbb{Z}^{n}}\left\|A_{Q_{0 k}} \vec{g}(k)\right\|^{p}\right)^{1 / p} \leq c_{p, n}\|\vec{g}\|_{L^{p}(W)} .
$$

Proof. Choose a scalar-valued function $\gamma \in \mathcal{S}$ with $\hat{\gamma}=1$ for $|\xi| \leq 2$ and supp $\hat{\gamma} \subseteq$ $\{|\xi|<\pi\}$. Then for $\vec{g} \in E_{0}$, we have $\vec{g}=\gamma * \vec{g}$, and the left-hand side of (6.2) is

$$
\begin{aligned}
& \sum_{k \in \mathbb{Z}^{n}} \int_{Q_{0 k}}\left\|W^{1 / p}(x) \int_{\mathbb{R}^{n}} \vec{g}(y) \gamma(k-y) d y\right\|^{p} d x \\
\leq & c_{M} \sum_{k \in \mathbb{Z}^{n}} \int_{Q_{0 k}}\left(\int_{\mathbb{R}^{n}} \frac{\left\|W^{1 / p}(x) \vec{g}(y)\right\|}{(1+|k-y|)^{M}} d y\right)^{p} d x,
\end{aligned}
$$

for some $M>n+\beta p / p^{\prime}$, where $\beta$ is the doubling exponent of $W$, since $\gamma \in \mathcal{S}$. Since $\mathbb{R}^{n}=\bigcup_{m \in \mathbb{Z}^{n}} Q_{0 m}$ and $m_{i} \leq y_{i}<m_{i}+1, i=1, \ldots, n$, on each $Q_{0 m}$, the last sum is bounded by

$$
c \sum_{k \in \mathbb{Z}^{n}} \int_{Q_{0 k}}\left(\sum_{m \in \mathbb{Z}^{n}} \frac{\int_{Q_{0 m}}\left\|W^{1 / p}(x) \vec{g}(y)\right\| d y}{(1+|k-m|)^{M}}\right)^{p} d x .
$$

Writing $M=M / p+M / p^{\prime}$ and using the discrete Hölder inequality (note that $M>n)$, we bound the last expression by

$$
c \sum_{k \in \mathbb{Z}^{n}} \int_{Q_{0 k}} \sum_{m \in \mathbb{Z}^{n}} \frac{\left(\int_{Q_{0 m}}\left\|W^{1 / p}(x) \vec{g}(y)\right\| d y\right)^{p}}{(1+|k-m|)^{M}} d x .
$$


Observe that

$$
\begin{gathered}
\left(\int_{Q_{0 m}}\left\|W^{1 / p}(x) \vec{g}(y)\right\| d y\right)^{p} \leq\left(\int_{Q_{0 m}}\left\|W^{1 / p}(x) W^{-1 / p}(y)\right\|\left\|W^{1 / p}(y) \vec{g}(y)\right\| d y\right)^{p} \\
\quad \leq\left(\int_{Q_{0 m}}\left\|W^{1 / p}(x) W^{-1 / p}(y)\right\|^{p^{\prime}} d y\right)^{p / p^{\prime}}\left(\int_{Q_{0 m}}\left\|W^{1 / p}(y) \vec{g}(y)\right\|^{p} d y\right)
\end{gathered}
$$

again by Hölder's inequality. By Lemma 4.3, $v_{x}(y)=\left\|W^{1 / p}(x) W^{-1 / p}(y)\right\|^{p^{\prime}}$ is a doubling measure with the doubling exponent $\beta$ :

$$
v_{x}\left(Q_{0 m}\right) \leq v_{x}(B(m,|k-m|+\sqrt{n})) \leq c(1+|k-m|)^{\beta} v_{x}\left(Q_{0 k}\right) .
$$

Thus, (6.4) is bounded by

$$
\begin{gathered}
c \sum_{k, m \in \mathbb{Z}^{n}}(1+|k-m|)^{\beta \frac{p}{p^{\prime}}-M}\left[\int_{Q_{0 k}}\left(\int_{Q_{0 k}}\left\|W^{1 / p}(x) W^{-1 / p}(y)\right\|^{p^{\prime}} d y\right)^{\frac{p}{p^{\prime}}} d x\right] \\
\times \int_{Q_{0 m}}\left\|W^{1 / p}(y) \vec{g}(y)\right\|^{p} d y .
\end{gathered}
$$

By Lemma 1.3, the expression in the square brackets of (6.5) is bounded by a constant independent of $k$. Since $M>\beta p / p^{\prime}+n$, the sum on $k$ converges and, therefore, (6.5) is estimated above by

$$
c \sum_{m \in \mathbb{Z}^{n}} \int_{Q_{0 m}}\left\|W^{1 / p}(y) \vec{g}(y)\right\|^{p} d y=c \int_{\mathbb{R}^{n}}\left\|W^{1 / p}(y) \vec{g}(y)\right\|^{p} d y=c\|\vec{g}\|_{L^{p}(W)}^{p} .
$$

Lemma 6.5. Let $W$ be a doubling matrix of order $p$ with doubling exponent $\beta$ such that $p>\beta$, and let $\vec{g} \in E_{0}$. Then (6.2) holds. Furthermore, if $W$ is a diagonal matrix, then (6.2) holds for any $1 \leq p<\infty$.

Proof. First, assume $(\vec{g})_{i} \in \mathcal{S}$ with supp $\hat{\vec{g}}_{i} \subseteq\{|\xi|<\pi\}, \quad i=1, \ldots, m$. We want to show that for such $\vec{g}$, the sum on the left-hand side of (6.2) is finite. Choosing $r>\beta+n$, we have

$$
\sum_{k \in \mathbb{Z}^{n}} \int_{Q_{0 k}}\left\|W^{1 / p}(x) \vec{g}(k)\right\|^{p} d x \leq \sum_{k \in \mathbb{Z}^{n}} \frac{c}{(1+|k|)^{r}} \int_{Q_{0 k}}\left\|W^{1 / p}(x)\right\|^{p} d x .
$$

Since $w(x)=\left\|W^{1 / p}(x)\right\|^{p}$ is a scalar doubling measure, $w\left(Q_{0 k}\right) \leq c(1+|k|)^{\beta} w\left(Q_{00}\right)$. Hence,

$$
\sum_{k \in \mathbb{Z}^{n}} \int_{Q_{0 k}}\left\|W^{1 / p}(x) \vec{g}(k)\right\|^{p} d x \leq \sum_{k \in \mathbb{Z}^{n}} \frac{c w\left(Q_{00}\right)}{(1+|k|)^{r-\beta}} \leq c w\left(Q_{00}\right)<\infty,
$$

since $r-\beta>n$.

Now we will prove (6.2) for $\vec{g}$ with $(\vec{g})_{i} \in S$ and supp $\hat{\vec{g}}_{i} \subseteq\{|\xi| \leq 3\}$, and then generalize it to $(\vec{g})_{i} \in S^{\prime}$. Let $0<\delta<1$. Then $B_{\delta}(k) \subseteq 3 Q_{0 k}$. Using the doubling property of $w_{k}(x)=\left\|W^{1 / p}(x) \vec{g}(k)\right\|^{p}$, we "squeeze" each $Q_{0 k}$ into $B_{\delta}(k)$ :

$$
w_{k}\left(Q_{0 k}\right) \leq w_{k}\left(3 Q_{0 k}\right) \leq c\left[\frac{\left|3 Q_{0 k}\right|}{\left|B_{\delta}(k)\right|}\right]^{\beta / n} w_{k}\left(B_{\delta}(k)\right) \leq c_{\beta} \delta^{-\beta} w_{k}\left(B_{\delta}(k)\right) .
$$


Hence, the left-hand side of (6.2) is bounded by

$$
c_{\beta} \delta^{-\beta} \sum_{k \in \mathbb{Z}^{n}} \int_{B_{\delta}(k)}\left\|W^{1 / p}(x) \vec{g}(k)\right\|^{p} d x .
$$

To estimate the integral, we will use the trivial identity $\vec{g}(k)=\vec{g}(x)+[\vec{g}(k)-\vec{g}(x)]$ for $x \in B_{\delta}(k)$. Apply the decomposition from Lemma 6.2 ( $\gamma$ is the same as in the previous lemma):

$$
\vec{g}(k)=\sum_{m \in \mathbb{Z}^{n}} \vec{g}(m) \gamma(k-m) \quad \text { and } \quad \vec{g}(x)=\sum_{m \in \mathbb{Z}^{n}} \vec{g}(m) \gamma(x-m) .
$$

Using the Mean Value Theorem for $[\gamma(k-m)-\gamma(x-m)]$ and the properties of $\gamma \in \mathcal{S}$ (note that $|x-k|<\delta$ ), we have

$$
\left\|W^{1 / p}(x) \vec{g}(k)\right\|^{p} \leq c_{p}\left\|W^{1 / p}(x) \vec{g}(x)\right\|^{p}+c_{p, M} \delta^{p} \sum_{m \in \mathbb{Z}^{n}} \frac{\left\|W^{1 / p}(x) \vec{g}(m)\right\|^{p}}{(1+|k-m|)^{M}}
$$

for some $M>\beta+n$. Integrating (6.7) over $B_{\delta}(k)$, we get

$$
\begin{gathered}
\int_{B_{\delta}(k)}\left\|W^{1 / p}(x) \vec{g}(k)\right\|^{p} d x \leq c_{p} \int_{B_{\delta}(k)}\left\|W^{1 / p}(x) \vec{g}(x)\right\|^{p} d x \\
+c \delta^{p} \sum_{m \in \mathbb{Z}^{n}} \frac{\int_{B_{\delta}(k)}\left\|W^{1 / p}(x) \vec{g}(m)\right\|^{p} d x}{(1+|k-m|)^{M}} .
\end{gathered}
$$

Apply the doubling property of $w_{m}(x)=\left\|W^{1 / p}(x) \vec{g}(m)\right\|^{p}$ again:

$$
\begin{gathered}
w_{m}\left(B_{\delta}(k)\right) \leq w_{m}(B(m,|k-m|+\delta)) \leq c\left[\frac{(\delta+|k-m|)^{n}}{\delta^{n}}\right]^{\beta / n} w_{m}\left(B_{\delta}(m)\right) \\
=c \delta^{-\beta}(1+|k-m|)^{\beta} w_{m}\left(B_{\delta}(m)\right) .
\end{gathered}
$$

Substituting this estimate into (6.8) and summing over $k \in \mathbb{Z}^{n}$, we have

$$
\begin{aligned}
& \sum_{k \in \mathbb{Z}^{n}} \int_{B_{\delta}(k)}\left\|W^{1 / p}(x) \vec{g}(k)\right\|^{p} d x \leq c_{p} \sum_{k \in \mathbb{Z}^{n}} \int_{B_{\delta}(k)}\left\|W^{1 / p}(x) \vec{g}(x)\right\|^{p} d x \\
+ & c \delta^{p-\beta} \sum_{m \in \mathbb{Z}^{n}} \int_{B_{\delta}(m)}\left\|W^{1 / p}(x) \vec{g}(m)\right\|^{p} d x\left(\sum_{k \in \mathbb{Z}^{n}}(1+|k-m|)^{\beta-M}\right),
\end{aligned}
$$

where the last sum converges since $M>\beta+n$. If $p>\beta$, by choosing $0<\delta<1 / 2$ such that $1-c \delta^{p-\beta}>0$, we subtract the last term from both sides (note that it is finite because of our estimates above for $\vec{g}_{i} \in \mathcal{S}$ ), substitute it into (6.6) and get the estimate of the left-hand side of (6.2) (note that $\sum_{k \in \mathbb{Z}^{n}} \int_{B_{\delta}(k)} \ldots \leq \int_{\mathbb{R}^{n}} \ldots$ ):

$$
\begin{gathered}
\sum_{k \in \mathbb{Z}^{n}} \int_{Q_{0 k}}\left\|W^{1 / p}(x) \vec{g}(k)\right\|^{p} d x \leq \frac{c_{\beta} \delta^{-\beta} c_{p}}{\left(1-c \delta^{p-\beta}\right)} \sum_{k \in \mathbb{Z}^{n}} \int_{B_{\delta}(k)}\left\|W^{1 / p}(x) \vec{g}(x)\right\|^{p} d x \\
\leq c_{n, \beta, p} \int_{\mathbb{R}^{n}}\left\|W^{1 / p}(x) \vec{g}(x)\right\|^{p} d x=c_{n, \beta, p}\|\vec{g}\|_{L^{p}(W)}^{p} .
\end{gathered}
$$

Now let $(\vec{g})_{i} \in S^{\prime}, i=1, \ldots, m$. Since $\vec{g} \in E_{0}$, it follows that $(\vec{g})_{i} \in C^{\infty}$, and $\vec{g}$ and all its derivatives are slowly increasing. Pick a scalar-valued $\gamma \in \mathcal{S}$ such that $\gamma(0)=1$ and supp $\hat{\gamma} \subseteq B(0,1)$. Then for $0<\epsilon<1$, the function $\vec{g}^{\epsilon}(x):=\vec{g}(x) \gamma(\epsilon x)$ has its 
components in $\mathcal{S}$. Observe that $\hat{\vec{g}}^{\epsilon}=\hat{\vec{g}} *[\gamma(\epsilon x)]^{\wedge}$, with $[\gamma(\epsilon x)]^{\wedge}(\xi)=(1 / \varepsilon) \hat{\gamma}(\xi / \varepsilon)$, and, therefore,

$$
\operatorname{supp} \hat{\vec{g}}^{\epsilon} \subseteq \operatorname{supp} \hat{\vec{g}}+\operatorname{supp}(1 / \varepsilon) \hat{\gamma}(\xi / \varepsilon) \subseteq\{\xi:|\xi|<3\} .
$$

We can apply the result (6.9) to $\vec{g}^{\epsilon}$ :

or

$$
\sum_{k \in \mathbb{Z}^{n}} \int_{Q_{0 k}}\left\|W^{1 / p}(x) \vec{g}^{\epsilon}(k)\right\|^{p} d x \leq c\left\|\vec{g}^{\epsilon}\right\|_{L^{p}(W)}^{p},
$$

$$
\sum_{k \in \mathbb{Z}^{n}} \int_{Q_{0 k}}\left\|W^{1 / p}(x) \vec{g}(k) \gamma(\epsilon k)\right\|^{p} d x \leq c \int_{\mathbb{R}^{n}}\left\|W^{1 / p}(x) \vec{g}(x)\right\|^{p}|\gamma(\epsilon x)|^{p} d x .
$$

Taking liminf as $\epsilon \rightarrow 0$ of both sides and using Fatou's Lemma on the left-hand side (with a discrete measure for the sum) and the Dominated Convergence Theorem on the right-hand side, we obtain

$$
\begin{aligned}
& \sum_{k \in \mathbb{Z}^{n}} \liminf _{\epsilon \rightarrow 0}|\gamma(\epsilon k)|^{p} \int_{Q_{0 k}}\left\|W^{1 / p}(x) \vec{g}(k)\right\|^{p} d x \\
& \leq c \int_{\mathbb{R}^{n}}\left\|W^{1 / p}(x) \vec{g}(x)\right\|^{p} \lim _{\epsilon \rightarrow 0}|\gamma(\epsilon x)|^{p} d x .
\end{aligned}
$$

Since $\gamma(\epsilon x) \underset{\epsilon \rightarrow 0}{\longrightarrow} \gamma(0)$, we obtain 6.2 for all $\vec{g} \in E_{0}$.

To get the second assertion of the lemma, we consider the scalar case with $w$ a scalar doubling measure. Then (6.8) becomes

$$
\begin{aligned}
& w\left(B_{\delta}(k)\right)|g(k)|^{p} \leq c_{p} \int_{B_{\delta}(k)} w(x)|g(x)|^{p} d x \\
& +c_{p} \delta^{p} w\left(B_{\delta}(k)\right) \sum_{m \in \mathbb{Z}^{n}} \frac{|g(m)|^{p}}{(1+|k-m|)^{M}}
\end{aligned}
$$

or

$$
|g(k)|^{p} \leq c_{p} \frac{1}{w\left(B_{\delta}(k)\right)} \int_{B_{\delta}(k)} w(x)|g(x)|^{p} d x+c \delta^{p} \sum_{m \in \mathbb{Z}^{n}} \frac{|g(m)|^{p}}{(1+|k-m|)^{M}} .
$$

We want to estimate the last sum on $m$. Fix $l \in \mathbb{Z}^{n}$. Dividing everything by $(1+|k-l|)^{M}$ and summing on $k \in \mathbb{Z}^{n}$, we get

$$
\begin{aligned}
& \sum_{k \in \mathbb{Z}^{n}} \frac{|g(k)|^{p}}{(1+|k-l|)^{M}} \leq c_{p} \sum_{k \in \mathbb{Z}^{n}} \frac{\int_{B_{\delta}(k)} w(x)|g(x)|^{p} d x}{(1+|k-l|)^{M} w\left(B_{\delta}(k)\right)} \\
& \quad+c \delta^{p} \sum_{k \in \mathbb{Z}^{n}} \frac{1}{(1+|k-l|)^{M}} \sum_{m \in \mathbb{Z}^{n}} \frac{|g(m)|^{p}}{(1+|k-m|)^{M}} .
\end{aligned}
$$

Note that in the last term

$$
\sum_{k \in \mathbb{Z}^{n}} \frac{1}{(1+|k-l|)^{M}(1+|k-m|)^{M}} \leq \frac{c}{(1+|l-m|)^{M}},
$$

since $M>n$. Therefore,

$$
\sum_{k \in \mathbb{Z}^{n}} \frac{|g(k)|^{p}}{(1+|k-l|)^{M}} \leq c_{p} \sum_{k \in \mathbb{Z}^{n}} \frac{\int_{B_{\delta}(k)} w(x)|g(x)|^{p} d x}{(1+|k-l|)^{M} w\left(B_{\delta}(k)\right)}+c \delta^{p} \sum_{m \in \mathbb{Z}^{n}} \frac{|g(m)|^{p}}{(1+|l-m|)^{M}} .
$$


Choose $0<\delta<1 / 2$ such that $1-c \delta^{p}>0$. Then

$$
\sum_{m \in \mathbb{Z}^{n}} \frac{|g(m)|^{p}}{(1+|l-m|)^{M}} \leq \frac{c_{p}}{1-c \delta^{p}} \sum_{m \in \mathbb{Z}^{n}} \frac{\int_{B_{\delta}(m)} w(x)|g(x)|^{p} d x}{(1+|l-m|)^{M} w\left(B_{\delta}(m)\right)} .
$$

Substituting this into (6.10) and summing on $k \in \mathbb{Z}^{n}$ (again using $\sum_{k \in \mathbb{Z}^{n}} \int_{B_{\delta}(k)} \cdots \leq$ $\left.\int_{\mathbb{R}^{n}} \cdots\right)$, we obtain

$$
\begin{aligned}
& \sum_{k \in \mathbb{Z}^{n}} w\left(B_{\delta}(k)\right)|g(k)|^{p} \\
& \quad \leq c_{p}\|g\|_{L^{p}(w)}^{p}+c \delta^{p} \sum_{k \in \mathbb{Z}^{n}} w\left(B_{\delta}(k)\right) \sum_{m \in \mathbb{Z}^{n}} \frac{\int_{B_{\delta}(m)} w(x)|g(x)|^{p} d x}{(1+|k-m|)^{M} w\left(B_{\delta}(m)\right)} .
\end{aligned}
$$

Use the doubling property of $w$ to shift $B_{\delta}(k)$ to $B_{\delta}(m)$. Since $\delta$ is fixed, $w\left(B_{\delta}(k)\right) \leq$ $c_{\delta, n}(1+|k-m|)^{\beta} w\left(B_{\delta}(m)\right)$, and thus, the last term is dominated by

$$
c \delta^{p} \sum_{m \in \mathbb{Z}^{n}} \int_{B_{\delta}(m)} w(x)|g(x)|^{p} d x \times\left(\sum_{k \in \mathbb{Z}^{n}}(1+|k-m|)^{\beta-M}\right),
$$

where the sum on $k$ converges, since $M>\beta+n$. Thus, (6.11) is estimated by $c_{p, n, \beta}\|g\|_{L^{p}(w)}^{p}$. Hence,

$$
\sum_{k \in \mathbb{Z}^{n}} \int_{Q_{0 k}} w(x)|g(k)|^{p} d x \leq c_{p, n, \beta} \sum_{k \in \mathbb{Z}^{n}} w\left(B_{\delta}(k)\right)|g(k)|^{p} \leq c_{p, n, \beta}\|g\|_{L^{p}(w)}^{p} .
$$

Now if $W$ is a diagonal matrix, then

$$
\left\|W^{1 / p}(x) \vec{u}\right\|^{p} \approx \sum_{i=1}^{m} w_{i i}(x)\left|\vec{u}_{i}\right|^{p},
$$

and thus, applying the scalar case, we get

$$
\begin{gathered}
\sum_{k \in \mathbb{Z}^{n}} \int_{Q_{0 k}}\left\|W^{1 / p}(x) \vec{g}(k)\right\|^{p} d x \approx \sum_{i=1}^{m} \sum_{k \in \mathbb{Z}^{n}} \int_{Q_{0 k}} w_{i i}(x)\left|\vec{g}_{i}(k)\right|^{p} d x \\
\leq \sum_{i=1}^{m} c\left\|\vec{g}_{i}\right\|_{L^{p}\left(w_{i i}\right)}^{p} \approx c_{p, n, \beta, m}\|\vec{g}\|_{L^{p}(W)}^{p} .
\end{gathered}
$$

Theorem 6.6. Let $\alpha \in \mathbb{R}, 0<q \leq \infty, 1 \leq p<\infty$, and let $W$ satisfy any of (A1)-(A3). Then

$$
\left\|\left\{\vec{s}_{Q}\right\}_{Q}\right\|_{\dot{b}_{p}^{\alpha q}(W)} \leq c\|\vec{f}\|_{\dot{B}_{p}^{\alpha q}(W)},
$$

where $\vec{s}_{Q}=S_{\varphi} \vec{f}=\left\langle\vec{f}, \varphi_{Q}\right\rangle$ for a given $\vec{f}$.

Proof. By definition,

$$
\left\|\left\{\vec{s}_{Q}\right\}_{Q}\right\|_{\dot{b}_{p}^{\alpha q}(W)}=\left\|\left\{\left\|\sum_{l(Q)=2^{-\nu}}|Q|^{-1 / 2}\right\| W^{1 / p} \cdot \vec{s}_{Q}\left\|_{\mathcal{H}} \chi_{Q}\right\|_{L^{p}}\right\}_{\nu}\right\|_{l_{q}^{\alpha}}=:\left\|\left\{J_{\nu}\right\}_{\nu}\right\|_{l_{q}^{\alpha}} .
$$


Fix $\nu \in \mathbb{Z}$. Then $Q=Q_{\nu k}=\prod_{i=1}^{n}\left[\frac{k_{i}}{2^{\nu}}, \frac{k_{i}+1}{2^{\nu}}\right], k \in \mathbb{Z}^{n},|Q|=2^{-\nu n}, \vec{s}_{Q}=$ $|Q|^{1 / 2}\left(\tilde{\varphi}_{\nu} * \vec{f}\right)\left(2^{-\nu} k\right)$ and

$$
\begin{aligned}
J_{\nu}^{p} & =\sum_{l(Q)=2^{-\nu}}|Q|^{-p / 2} \int_{Q}\left\|W^{1 / p}(t) \vec{s}_{Q}\right\|^{p} d t \\
& =\sum_{k \in \mathbb{Z}^{n}} \int_{Q_{\nu k}}\left\|W^{1 / p}(t)\left(\tilde{\varphi}_{\nu} * \vec{f}\right)\left(2^{-\nu} k\right)\right\|^{p} d t .
\end{aligned}
$$

Let $\vec{f}_{\nu}(x)=\vec{f}\left(2^{-\nu} x\right)$. Then $\left(\tilde{\varphi}_{\nu} * \vec{f}\right)\left(2^{-\nu} k\right)=\left(\tilde{\varphi} * \vec{f}_{\nu}\right)(k)$. We substitute this in the last integral and note that the change of variables $y=2^{\nu} t$ (with $W_{\nu}(t):=W\left(2^{-\nu} t\right)$ ) will yield

$$
J_{\nu}^{p}=2^{-\nu n} \sum_{k \in \mathbb{Z}^{n}} \int_{Q_{0 k}}\left\|W_{\nu}^{1 / p}(t)\left(\tilde{\varphi} * \vec{f}_{\nu}\right)(k)\right\|^{p} d t .
$$

Observe that $\left(\tilde{\varphi} * \vec{f}_{\nu}\right)_{i} \in \mathcal{S}^{\prime}, i=1, \ldots, m$, and $\tilde{\varphi} * \vec{f}_{\nu} \in E_{0}$, since supp $\hat{\tilde{\varphi}} \subseteq\{\xi \in$ $\left.\mathbb{R}^{n}: \frac{1}{2} \leq|\xi| \leq 2\right\}$. Using either Lemma 6.3 or Lemma 6.5 with $\vec{g}=\tilde{\varphi} * \vec{f}_{\nu}$ and $W_{\nu}$ instead of $W$ (both the $A_{p}$ condition and the doubling condition are invariant with respect to dilation), we obtain

$$
J_{\nu}^{p} \leq c 2^{-\nu n} \int_{\mathbb{R}^{n}}\left\|W_{\nu}^{1 / p}(t)\left(\tilde{\varphi} * \vec{f}_{\nu}\right)(t)\right\|^{p} d t .
$$

Changing variables, we get

$$
J_{\nu}^{p} \leq c \int_{\mathbb{R}^{n}}\left\|W^{1 / p}(t)\left(\tilde{\varphi}_{\nu} * \vec{f}\right)(t)\right\|^{p} d t=c\left\|\left(\tilde{\varphi}_{\nu} * \vec{f}\right)\right\|_{L^{p}(W)}^{p} .
$$

Combining the estimates of $J_{\nu}$ for all $\nu$ into (6.13), we get

$$
\begin{gathered}
\left\|\left\{\left\langle\vec{f}, \varphi_{Q}\right\rangle\right\}_{Q}\right\|_{\dot{b}_{p}^{\alpha q}(W)}=\left\|\left\{\vec{s}_{Q}\right\}_{Q}\right\|_{\dot{b}_{p}^{\alpha q}(W)}=\left\|\left\{J_{\nu}\right\}_{\nu}\right\|_{l_{q}^{\alpha}} \\
\leq c\left\|\left\{\left\|\left(\tilde{\varphi}_{\nu} * \vec{f}\right)\right\|_{L^{p}(W)}\right\}_{\nu}\right\|_{l_{q}^{\alpha}}=c\|\vec{f}\|_{\dot{B}_{p}^{\alpha q}(W, \tilde{\varphi})},
\end{gathered}
$$

where $c=c(p, \beta, n)$.

To finish the proof of the theorem, we have to establish the equivalence between $\dot{B}_{p}^{\alpha q}(W, \varphi)$ and $\dot{B}_{p}^{\alpha q}(W, \tilde{\varphi})$. As we mentioned in Section $2, \tilde{\varphi} \in \mathcal{A}$, and so the pair $(\tilde{\varphi}, \tilde{\psi})$ satisfies (2.1), since $\hat{\tilde{\varphi}}=\overline{\hat{\varphi}}$ and $\hat{\tilde{\psi}}=\overline{\hat{\psi}}$. By (2.2), $\vec{f}=\sum_{Q}\left\langle\vec{f}, \tilde{\varphi}_{Q}\right\rangle \tilde{\psi}_{Q}$. Since

$\left\{\tilde{\psi}_{Q}\right\}_{Q}$ is a family of smooth molecules for $\dot{B}_{p}^{\alpha q}(W)$ (see Remark [5.5), by Theorem 5.2 we have

$$
\|\vec{f}\|_{\dot{B}_{p}^{\alpha q}(W, \tilde{\varphi})} \leq c\left\|\left\{\left\langle\vec{f}, \tilde{\varphi}_{Q}\right\rangle\right\}_{Q}\right\|_{\dot{b}_{p}^{\alpha q}(W)} .
$$

Applying (6.15) to the right-hand side of the last inequality, we bound it by

$$
c\|\vec{f}\|_{\dot{B}_{p}^{\alpha q}(W, \tilde{\varphi})}=c\|\vec{f}\|_{\dot{B}_{p}^{\alpha q}(W, \varphi)} .
$$


Finally, combining (6.15) with (6.16) and (6.17), we obtain

$$
\left\|\left\{\left\langle\vec{f}, \varphi_{Q}\right\rangle\right\}_{Q}\right\|_{\dot{b}_{p}^{\alpha q}(W)} \equiv\left\|\left\{\vec{s}_{Q}\right\}_{Q}\right\|_{\dot{b}_{p}^{\alpha q}(W)} \leq c\|\vec{f}\|_{\dot{B}_{p}^{\alpha q}(W, \varphi)} .
$$

Remark 6.7. The fact that $\varphi$ and $\tilde{\varphi}$ were interchanged in the last step of the previous theorem can be generalized into Theorem 1.8 about the independence of the space $\dot{B}_{p}^{\alpha q}(W)$ from the choice of $\varphi$ :

Proof of Theorem 1.8, Let $\left\{\varphi^{(1)}, \psi^{(1)}\right\}$ and $\left\{\varphi^{(2)}, \psi^{(2)}\right\}$ be two different sets of mutually admissible kernels. Decompose $\vec{f}$ in the second system:

$$
\vec{f}=\sum_{Q}\left\langle\vec{f}, \varphi_{Q}^{(2)}\right\rangle \psi_{Q}^{(2)}=\sum_{Q} \vec{s}_{Q}^{(2)} \psi_{Q}^{(2)}
$$

Observe that $\psi_{Q}^{(2)}$ is a molecule for $Q$ and, therefore, by Theorem 5.2

$$
\|\vec{f}\|_{\dot{B}_{p}^{\alpha q}\left(W, \varphi^{(1)}\right)} \leq c\left\|\left\{\vec{s}_{Q}^{(2)}\right\}_{Q}\right\|_{\dot{b}_{p}^{\alpha q}(W)} \leq c\|\vec{f}\|_{\dot{B}_{p}^{\alpha q}\left(W, \varphi^{(2)}\right)},
$$

where the last inequality holds by Theorem 6.6 Interchanging $\varphi^{(1)}$ with $\varphi^{(2)}$, we get the norm equivalence between $\dot{B}_{p}^{\alpha q}\left(W, \varphi^{(1)}\right)$ and $\dot{B}_{p}^{\alpha q}\left(W, \varphi^{(2)}\right)$. In other words, the space $\dot{B}_{p}^{\alpha q}(W)$ is independent of the choice of $\varphi$ under any of the three assumptions on $W$.

Remark 6.8. Combining boundedness of the $\varphi$-transform (Theorem 6.6) and that of the inverse $\varphi$-transform (Corollary 5.6), we get the norm equivalence claimed in Theorems 1.4 and 1.6

\section{Connection With Reducing operators}

Now we connect the weighted sequence Besov space with its reducing operator equivalent. Recall that for each matrix weight $W$, we can find a sequence of reducing operators $\left\{A_{Q}\right\}_{Q}$ such that

$$
\rho_{p, Q}(\vec{u})=\left(\frac{1}{|Q|} \int\left\|W^{1 / p}(t) \cdot \vec{u}\right\|_{\mathcal{H}}^{p} \chi_{Q}(t) d t\right)^{1 / p} \approx\left\|A_{Q} \vec{u}\right\|_{\mathcal{H}}, \text { for all } \vec{u} \in \mathcal{H}
$$

Lemma 7.1. Let $\alpha \in \mathbb{R}, 0<q \leq \infty, 1 \leq p<\infty$, and let $\left\{A_{Q}\right\}_{Q}$ be reducing operators for $W$. Then

$$
\left\|\left\{\vec{s}_{Q}\right\}_{Q}\right\|_{\dot{b}_{p}^{\alpha q}(W)} \approx\left\|\left\{\vec{s}_{Q}\right\}_{Q}\right\|_{\dot{b}_{p}^{\alpha q}\left(\left\{A_{Q}\right\}\right)} .
$$


Proof. Using (7.1), we get the equivalence

$$
\begin{aligned}
\left\|\left\{\vec{s}_{Q}\right\}_{Q}\right\|_{\dot{b}_{p}^{\alpha q}(W)} & =\left\|\left\{\left\|\sum_{l(Q)=2^{-\nu}}|Q|^{-\frac{1}{2}}\right\| W^{1 / p} \cdot \vec{s}_{Q}\left\|_{\mathcal{H}} \chi_{Q}\right\|_{L^{p}}\right\}_{\nu}\right\|_{l_{q}^{\alpha}} \\
& =\left\|\left\{\left(\sum_{l(Q)=2^{-\nu}}|Q|^{-\frac{p}{2}}\left[\rho_{p, Q}\left(\vec{s}_{Q}\right)\right]^{p}|Q|\right)^{\frac{1}{p}}\right\}_{\nu}\right\|_{l_{q}^{\alpha}} \\
& \approx\left\|\left\{\left(\sum_{l(Q)=2^{-\nu}}|Q|^{-\frac{p}{2}}\left\|A_{Q} \vec{s}_{Q}\right\|_{\mathcal{H}}^{p} \int \chi_{Q}(t) d t\right)^{\frac{1}{p}}\right\}_{\nu}\right\|_{l_{q}^{\alpha}} \\
& =\left\|\left\{\left\|\sum_{l(Q)=2^{-\nu}}|Q|^{-\frac{1}{2}}\right\| A_{Q} \vec{s}_{Q}\left\|_{\mathcal{H}} \chi_{Q}\right\|_{L^{p}}\right\}_{\nu}\right\|_{l_{q}^{\alpha}} \\
& =\left\|\left\{\vec{s}_{Q}\right\}_{Q}\right\|_{\dot{b}_{p}^{\alpha q}\left(\left\{A_{Q}\right\}\right)^{\circ}}
\end{aligned}
$$

Finally, combining Theorems 1.4 and 1.6 with (7.2), we get Theorem 1.9 .

Corollary 7.2. The space $\dot{B}_{p}^{\alpha q}(W)$ is complete when $\alpha \in \mathbb{R}, 0<q \leq \infty, 1 \leq p<$ $\infty$ and $W$ satisfies any of (A1)-(A3).

Proof. If $\left\{\vec{f}_{n}\right\}_{n \in \mathbb{N}}$ is Cauchy in $\dot{B}_{p}^{\alpha q}(W)$, then $\left\{\left\{\vec{s}_{Q}\left(\vec{f}_{n}\right)\right\}_{Q}\right\}_{n \in \mathbb{N}}$ is Cauchy in $\dot{b}_{p}^{\alpha q}\left(\left\{A_{Q}\right\}\right)$ by Theorem 6.6 and Lemma 7.1 (or just Theorem [1.9). This implies that

$$
\begin{gathered}
\left.\left\|\sum_{l(Q)=2^{-\nu}}|Q|^{-\frac{1}{2}}\right\| A_{Q}\left[\vec{s}_{Q}\left(\vec{f}_{n}\right)-\vec{s}_{Q}\left(\vec{f}_{m}\right)\right]\right]\left\|_{\mathcal{H}} \chi_{Q}\right\|_{L^{p}}^{p} \\
=2^{\nu n(p / 2-1)} \sum_{l(Q)=2^{-\nu}}\left\|A_{Q}\left[\vec{s}_{Q}\left(\vec{f}_{n}\right)-\vec{s}_{Q}\left(\vec{f}_{m}\right)\right]\right\|_{\mathcal{H}}^{p} \underset{n, m \rightarrow \infty}{\longrightarrow} 0, \text { for each } \nu \in \mathbb{Z} .
\end{gathered}
$$

Hence, $\left\|A_{Q}\left[\vec{s}_{Q}\left(\vec{f}_{n}\right)-\vec{s}_{Q}\left(\vec{f}_{m}\right)\right]\right\|_{\mathcal{H}} \underset{n, m \rightarrow \infty}{\longrightarrow} 0$ for each $Q$. Since the $A_{Q}$ 's are invertible, $\left\{\vec{s}_{Q}\left(\vec{f}_{n}\right)\right\}_{n \in \mathbb{N}}$ is a vector-valued Cauchy sequence in $\mathcal{H}$ for each $Q$. Therefore, we can define $\vec{s}_{Q}=\lim _{n \rightarrow \infty} \vec{s}_{Q}\left(\vec{f}_{n}\right)$. Set $\vec{f}=\sum_{Q} \vec{s}_{Q} \psi_{Q}$. Observe that

$$
\begin{aligned}
\left\|\vec{f}_{n}-\vec{f}\right\|_{\dot{B}_{p}^{\alpha q}(W)} & =\left\|\sum_{Q}\left[\vec{s}_{Q}\left(\vec{f}_{n}\right)-\vec{s}_{Q}\right] \psi_{Q}\right\|_{\dot{B}_{p}^{\alpha q}(W)} \\
& \leq c\left\|\left\{\vec{s}_{Q}\left(\vec{f}_{n}\right)-\vec{s}_{Q}\right\}_{Q}\right\|_{\dot{b}_{p}^{\alpha q}\left(\left\{A_{Q}\right\}\right)} \\
& \leq c \liminf _{m \rightarrow \infty}\left\|\left\{\vec{s}_{Q}\left(\vec{f}_{n}\right)-\vec{s}_{Q}\left(\vec{f}_{m}\right)\right\}_{Q}\right\|_{\dot{b}_{p}^{\alpha q}\left(\left\{A_{Q}\right\}\right)} \underset{n \rightarrow \infty}{\longrightarrow} 0,
\end{aligned}
$$


by Corollary 5.6 and Lemma 7.1 , the discrete version of Fatou's Lemma and the fact that $\left\{\left\{\vec{s}_{Q}\left(\overrightarrow{f_{n}}\right)\right\}_{Q}\right\}_{n \in \mathbb{N}}$ is Cauchy in $\dot{b}_{p}^{\alpha q}\left(\left\{A_{Q}\right\}\right)$. Furthermore, $\vec{f}=\left(\vec{f}-\vec{f}_{n}\right)+\vec{f}_{n} \in$ $\dot{B}_{p}^{\alpha q}(W)$. Thus, $\dot{B}_{p}^{\alpha q}(W)$ is complete.

Recall the $A_{p}$ condition in terms of reducing operators: $\left\|A_{Q} A_{Q}^{\#}\right\| \leq c$ for any cube $Q \in \mathbb{R}^{n}$; in other words, $\left\|A_{Q} y\right\| \leq c\left\|\left(A_{Q}^{\#}\right)^{-1} y\right\|$ holds for any $y \in \mathcal{H}$. Note that the inverse inequality $\left\|\left(A_{Q} A_{Q}^{\#}\right)^{-1}\right\| \leq c$ (or, equivalently, $\left\|\left(A_{Q}^{\#}\right)^{-1} y\right\| \leq c\left\|A_{Q} y\right\|$ for any $y \in \mathcal{H}$ ) holds automatically (a straightforward application of Hölder's inequality). This implies the following imbeddings of the sequence Besov spaces:

Corollary 7.3. For $\alpha \in \mathbb{R}, 1<p<\infty, 0<q \leq \infty$, and $W$ a matrix weight with corresponding reducing operators $A_{Q}$ and $A_{Q}^{\#}$,

1. $\dot{b}_{p}^{\alpha q}\left(\left\{A_{Q}\right\}\right) \subseteq \dot{b}_{p}^{\alpha q}\left(\left\{\left(A_{Q}^{\#}\right)^{-1}\right\}\right)$ always, and

2. $\dot{b}_{p}^{\alpha q}\left(\left\{\left(A_{Q}^{\#}\right)^{-1}\right\}\right) \subseteq \dot{b}_{p}^{\alpha q}\left(\left\{A_{Q}\right\}\right)$ if $W \in A_{p}$.

\section{Almost diagonal operators}

Consider $\dot{b}_{p}^{\alpha q}(W)$ with parameters $\alpha, p, q$ fixed $(\alpha \in \mathbb{R}, 1 \leq p<\infty, 0<q \leq \infty)$, and $W$ a doubling matrix of order $p$ with doubling exponent $\beta$. If $p=1$, then $1 / p^{\prime}=0$.

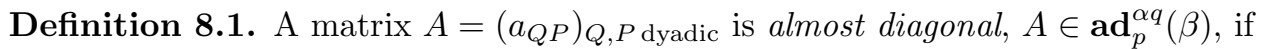
there exist $M>J=\frac{n}{p^{\prime}}+\frac{\beta}{p}$ and $c>0$ such that for all $Q, P$,

$$
\left|a_{Q P}\right| \leq c \min \left(\left[\frac{l(Q)}{l(P)}\right]^{\alpha_{1}},\left[\frac{l(P)}{l(Q)}\right]^{\alpha_{2}}\right)\left(1+\frac{\left|x_{Q}-x_{P}\right|}{\max (l(Q), l(P))}\right)^{-M}
$$

with $\alpha_{1}>\alpha+\frac{n}{2}$ and $\alpha_{2}>J-\left(\alpha+\frac{n}{2}\right)$.

Remark 8.2. This definition differs from the definition of almost diagonality in [3], since both $\alpha_{2}$ and $M$ depend on the doubling exponent $\beta$.

To simplify notation for the matrix $A$ above, we will only write $\left(a_{Q P}\right)$ without specifying indices $Q, P$.

Example 8.3 (An ALmost Diagonal matrix). Let $\varphi \in \mathcal{A}$. If $\left\{m_{Q}\right\}_{Q}$ is a family of smooth molecules for $\dot{B}_{p}^{\alpha q}(W)$, then

$$
\left(a_{Q P}\right) \in \mathbf{a d}_{p}^{\alpha q}(\beta)
$$

where $a_{Q P}=\left\langle m_{P}, \varphi_{Q}\right\rangle$, by (5.2) and (5.3), since $\left\langle m_{P}, \varphi_{Q}\right\rangle=|Q|^{1 / 2}\left(\tilde{\varphi}_{\nu} * m_{P}\right)\left(x_{Q}\right)$ if $l(Q)=2^{-\nu}$.

Now we show that almost diagonal matrices are bounded on $\dot{b}_{p}^{\alpha q}(W)$, i.e., Theorem 1.10. First we need the following approximation lemma, whose proof is trivial: 
Lemma 8.4. Let $P, Q$ be dyadic cubes and $t \in Q$. Then

$$
1+\frac{\left|x_{Q}-x_{P}\right|}{\max (l(Q), l(P))} \underset{(n)}{\approx} 1+\frac{\left|t-x_{P}\right|}{\max (l(Q), l(P))} .
$$

Proof of Theorem 1.10. Let $A=\left(a_{Q P}\right)$ with $A \in \mathbf{a d}_{p}^{\alpha q}(\beta)$. We want to show that

$$
\left\|\left\{\sum_{P} a_{Q P} \vec{s}_{P}\right\}_{Q}\right\|_{\dot{b}_{p}^{\alpha q}(W)} \leq c_{n, p, q, \beta}\left\|\left\{\vec{s}_{Q}\right\}_{Q}\right\|_{\dot{b}_{p}^{\alpha q}(W)} .
$$

By definition,

$$
\begin{aligned}
& \left\|\left\{\sum_{P} a_{Q P} \vec{s}_{P}\right\}_{Q}\right\|_{b_{p}^{\alpha q}(W)} \\
& \leq\left\|\left\{\left(\sum_{l(Q)=2^{-\nu}}|Q|^{-p / 2} \int_{Q}\left(\sum_{P}\left|a_{Q P}\right|\left\|W^{1 / p}(t) \vec{s}_{P}\right\|\right)^{p} d t\right)^{1 / p}\right\}_{\nu}\right\|_{l_{q}^{\alpha}} \\
& =:\left\|\left\{2^{\nu \alpha} 2^{\nu n / 2}\left(\sum_{l(Q)=2^{-\nu}} J_{Q}\right)^{1 / p}\right\}_{\nu}\right\|_{l^{q}} .
\end{aligned}
$$

Substituting the estimate (8.1) for $a_{Q P}$ in $J_{Q}$, we get

$$
\begin{aligned}
& J_{Q} \leq c_{p, M} \int_{Q}\left(\sum_{j \geq 0} 2^{-j \alpha_{2}} \sum_{l(P)=2^{-(\nu+j)}}\left\|W^{1 / p}(t) \vec{s}_{P}\right\|\left(1+2^{\nu}\left|x_{Q}-x_{P}\right|\right)^{-M}\right)^{p} d t \\
& +c_{p, M} \int_{Q}\left(\sum_{j<0} 2^{j \alpha_{1}} \sum_{l(P)=2^{-(\nu+j)}}\left\|W^{1 / p}(t) \vec{s}_{P}\right\|\left(1+2^{(\nu+j)}\left|x_{Q}-x_{P}\right|\right)^{-M}\right)^{p} d t .
\end{aligned}
$$

Pick $\epsilon>0$ sufficiently small such that (i) $\alpha_{1}-\epsilon>\alpha+n / 2$, (ii) $\alpha_{2}-\epsilon>J-\alpha-n / 2$ and (iii) $M>\beta / p+(n+\epsilon) / p^{\prime}$. Apply the discrete Hölder inequality twice, first with $\alpha_{i}=\epsilon+\left(\alpha_{i}-\epsilon\right)$ for the sum on $j$ (note that $\alpha_{1}, \alpha_{2}>0$ ) and second with 
$M=\frac{n+\epsilon}{p^{\prime}}+\left(M-\frac{n+\epsilon}{p^{\prime}}\right)$ for the sum on $P$ :

$$
\begin{aligned}
J_{Q} \leq c_{p, M} & \int_{Q}\left(\sum_{j \geq 0} 2^{-j \epsilon p^{\prime}}\right)^{p / p^{\prime}}\left[\sum_{j \geq 0} 2^{-j\left(\alpha_{2}-\epsilon\right) p}\right. \\
& \left.\times\left(\sum_{l(P)=2^{-(\nu+j)}}\left\|W^{1 / p}(t) \vec{s}_{P}\right\|\left(1+2^{\nu}\left|x_{Q}-x_{P}\right|\right)^{-M}\right)^{p}\right] d t \\
& +c_{p, M} \int_{Q}\left(\sum_{j<0} 2^{j \epsilon p^{\prime}}\right)^{p / p^{\prime}}\left[\sum_{j<0} 2^{j\left(\alpha_{1}-\epsilon\right) p}\right. \\
& \left.\times\left(\sum_{l(P)=2^{-(\nu+j)}}\left\|W^{1 / p}(t) \vec{s}_{P}\right\|\left(1+2^{(\nu+j)}\left|x_{Q}-x_{P}\right|\right)^{-M}\right)^{p}\right] d t \\
\leq & c_{p, M, \epsilon} \sum_{j \geq 0} 2^{-j\left(\alpha_{2}-\epsilon\right) p}\left[\sum_{l(P)=2^{-(\nu+j)}}\left(1+2^{\nu}\left|x_{Q}-x_{P}\right|\right)^{-n-\epsilon}\right]^{p / p^{\prime}} \\
& \times \sum_{l(P)=2^{-(\nu+j)}} \int_{Q}\left\|W^{1 / p}(t) \vec{s}_{P}\right\|^{p}\left(1+2^{\nu}\left|x_{Q}-x_{P}\right|\right)^{-\left(M-\frac{n+\epsilon}{p^{\prime}}\right) p} d t \\
& +c_{p, M, \epsilon} \sum_{j<0} 2^{j\left(\alpha_{1}-\epsilon\right) p}\left[\sum_{l(P)=2^{-(\nu+j)}}\left(1+2^{(\nu+j)}\left|x_{Q}-x_{P}\right|\right)^{-n-\epsilon}\right]^{p / p^{\prime}} \\
& \times \sum_{l(P)=2^{-(\nu+j)}} \int_{Q}\left\|W^{1 / p}(t) \vec{s}_{P}\right\|^{p}\left(1+2^{(\nu+j)}\left|x_{Q}-x_{P}\right|\right)^{-\left(M-\frac{n+\epsilon}{p^{\prime}}\right) p} d t .
\end{aligned}
$$

Use the Summation Lemma 5.4 to estimate the square brackets and denote $w_{P}(t)=$ $\left\|W^{1 / p}(t) \vec{s}_{P}\right\|^{p}$. By Lemma 8.4, $x_{Q}$ can be replaced by any $t \in Q$, and so we get

$$
\begin{aligned}
J_{Q} \leq & c_{p, M} \sum_{j \geq 0} 2^{-j\left(\alpha_{2}-\epsilon\right) p+j n p / p^{\prime}} \\
& \times \sum_{l(P)=2^{-(\nu+j)}} \int_{Q} w_{P}(t)\left(1+\frac{\left|t-x_{P}\right|}{l(Q)}\right)^{-\left(M-\frac{n+\epsilon}{p^{\prime}}\right) p} d t \\
& +c_{p, M} \sum_{j<0} 2^{j\left(\alpha_{1}-\epsilon\right) p} \sum_{l(P)=2^{-(\nu+j)}} \int_{Q} w_{P}(t)\left(1+\frac{\left|t-x_{P}\right|}{l(P)}\right)^{-\left(M-\frac{n+\epsilon}{p^{\prime}}\right) p} d t .
\end{aligned}
$$


Summing on $Q$ and applying the Squeeze Lemma 5.3 (recall $\left.M>\beta / p+(n+\epsilon) / p^{\prime}\right)$, we get

$$
\begin{aligned}
& \sum_{l(Q)=2^{-\nu}} J_{Q} \leq c_{p, n} \sum_{j \geq 0} 2^{-j\left(\alpha_{2}-\epsilon\right) p+j n p / p^{\prime}} \\
& \times \sum_{l(P)=2^{-(\nu+j)}} \sum_{l(Q)=2^{-\nu}} \int_{Q} w_{P}(t)\left(1+2^{\nu}\left|t-x_{P}\right|\right)^{-\left(M-\frac{n+\epsilon}{p^{\prime}}\right) p} d t \\
& +c_{p, n} \sum_{j<0} 2^{j\left(\alpha_{1}-\epsilon\right) p} \sum_{l(P)=2^{-(\nu+j)}} \sum_{l(Q)=2^{-\nu}} \int_{Q} w_{P}(t)\left(1+2^{\nu+j}\left|t-x_{P}\right|\right)^{-\left(M-\frac{n+\epsilon}{p^{\prime}}\right) p} d t \\
& \leq c_{p, n, \beta} \sum_{j \in \mathbb{Z}}\left(2^{-j\left(\alpha_{2}-\epsilon\right) p+j n p / p^{\prime}+j \beta} \chi_{\{j \geq 0\}}+2^{j\left(\alpha_{1}-\epsilon\right) p} \chi_{\{j<0\}} \sum_{l(P)=2^{-(\nu+j)}} w_{P}(P) .\right.
\end{aligned}
$$

Observe that $2^{\nu n p / 2}=|P|^{-p / 2} 2^{-j n p / 2}$ for $l(P)=2^{-(\nu+j)}$, and

$$
\sum_{l(P)=2^{-(\nu+j)}}|P|^{-p / 2} w_{P}(P)=\left\|\sum_{l(P)=2^{-(\nu+j)}}|P|^{-1 / 2} \vec{s}_{P} \chi_{P}\right\|_{L^{p}(W)}^{p} .
$$

Then, using $1<p<\infty$ to take the power $1 / p$ inside the sum on $j$, we get

$$
\begin{gathered}
2^{\nu \alpha} 2^{\nu n / 2}\left(\sum_{l(Q)=2^{-\nu}} J_{Q}\right)^{1 / p} \leq c \sum_{j \in \mathbb{Z}}\left[2 ^ { - j \alpha } 2 ^ { - j n / 2 } \left(2^{-j\left(\alpha_{2}-\epsilon\right) p+j n p / p^{\prime}+j \beta} \chi_{\{j \geq 0\}}\right.\right. \\
\left.\left.+2^{j\left(\alpha_{1}-\epsilon\right) p} \chi_{\{j<0\}}\right)^{1 / p}\right] \times 2^{(\nu+j) \alpha}\left\|\sum_{l(P)=2^{-(\nu+j)}}|P|^{-1 / 2} \vec{s}_{P} \chi_{P}\right\|_{L^{p}(W)} \\
=: c \sum_{j \in \mathbb{Z}} a_{-j} \times b_{\nu+j}=c(a * b)(\nu) .
\end{gathered}
$$

Use (5.10) and (5.11) to estimate the norm of the convolution $\|a * b\|_{l^{q}}$. Then for $q \leq 1$

$$
\|a\|_{l^{q}}^{q}=\sum_{j \leq 0} 2^{j\left(\alpha+n / 2+\left(\alpha_{2}-\epsilon\right)-J\right) q}+\sum_{j>0} 2^{j\left(\alpha+n / 2-\left(\alpha_{1}-\epsilon\right)\right) q} \leq c_{q}
$$

since $\alpha_{1}-\epsilon>\alpha+n / 2$ and $\alpha_{2}-\epsilon>J-(\alpha+n / 2)$. Using the $\|a\|_{l^{1}}$ estimate for $q \geq 1$, and the $\|a\|_{l^{q}}$ estimate for $q<1$, and substituting into (8), we obtain

$$
\begin{aligned}
\left\|\left\{\sum_{P} a_{Q P} \vec{s}_{P}\right\}_{Q}\right\|_{\dot{b}_{P}^{\alpha q}(W)} & \leq c\|b\|_{l^{q}} \\
& =c\left\|\left\{2^{\mu \alpha}\left\|\sum_{l(P)=2^{-\mu}}|P|^{-1 / 2} \vec{s}_{P} \chi_{P}\right\|_{L^{p}(W)}\right\}_{\mu}\right\|_{l^{q}} \\
& =c\left\|\left\{\vec{s}_{P}\right\}_{P}\right\|_{\dot{b}_{P}^{\alpha q}(W)},
\end{aligned}
$$

where $c=c_{n, p, q, \beta}$. 
Now we will show that the class of almost diagonal matrices is closed under composition. For $\epsilon>0, \delta>0, J=\frac{n}{p^{\prime}}+\frac{\beta}{p}$ and $P, Q$ dyadic, denote

$$
\begin{aligned}
& w_{Q P}(\delta, \epsilon) \\
& =\left[\frac{l(Q)}{l(P)}\right]^{\alpha+\frac{n}{2}} \min \left(\left[\frac{l(Q)}{l(P)}\right]^{\frac{\epsilon}{2}},\left[\frac{l(P)}{l(Q)}\right]^{\frac{\epsilon}{2}+J}\right)\left(1+\frac{\left|x_{Q}-x_{P}\right|}{\max (l(Q), l(P))}\right)^{-J-\delta} .
\end{aligned}
$$

Theorem 8.5. Let $A, B \in \operatorname{ad}_{p}^{\alpha q}(\beta)$. Then $A \circ B \in \operatorname{ad}_{p}^{\alpha q}(\beta)$.

We need the following lemma, which is a modification of Theorem D.2 in [5] adjusted to the weighted ad condition:

Lemma 8.6. Let $\delta, \gamma_{1}, \gamma_{2}>0, \gamma_{1} \neq \gamma_{2}$, and $2 \delta<\gamma_{1}+\gamma_{2}$. Then there exists a constant $c=c_{n, \delta, \gamma_{1}, \gamma_{2}, J}$ such that

$$
\sum_{R} w_{Q R}\left(\delta, \gamma_{1}\right) w_{R P}\left(\delta, \gamma_{2}\right) \leq c w_{Q P}\left(\delta, \min \left(\gamma_{1}, \gamma_{2}\right)\right) .
$$

Proof of Theorem 8.5. Since $A=\left(a_{Q P}\right), B=\left(b_{Q P}\right) \in \mathbf{a d}_{p}^{\alpha q}(\beta)$, for each $i=A, B$ there exist $0<\epsilon_{i}<\min \left(\alpha_{1}-(\alpha+n / 2), \alpha_{2}-J+\alpha+n / 2\right)$ and $0<\delta<M-J$ such that $\left|a_{Q P}\right| \leq c w_{Q P}\left(\delta, \epsilon_{A}\right)$ and $\left|b_{Q P}\right| \leq c w_{Q P}\left(\delta, \epsilon_{B}\right)$. Without loss of generality, we may assume $\epsilon_{A}<\epsilon_{B}$ and $\delta<\frac{\epsilon_{A}+\epsilon_{B}}{2}$. Then

$$
\left|(A B)_{Q P}\right| \leq\left|\sum_{R} a_{Q R} b_{R P}\right| \leq c \sum_{R} w_{Q R}\left(\delta, \epsilon_{A}\right) w_{R P}\left(\delta, \epsilon_{B}\right) \leq c w_{Q P}\left(\delta, \epsilon_{A}\right),
$$

by Lemma 8.6, which means that $A \circ B \in \operatorname{ad}_{p}^{\alpha q}(\beta)$.

Definition 8.7. Let $T$ be a continuous linear operator from $\mathcal{S}$ to $\mathcal{S}^{\prime}$. We say that $T$ is an almost diagonal operator for $\dot{B}_{p}^{\alpha q}(W)$, and write $T \in \mathbf{A D}_{p}^{\alpha q}(\beta)$, if for some pair of mutually admissible kernels $(\varphi, \psi)$, the matrix $\left(a_{Q P}\right) \in \operatorname{ad}_{p}^{\alpha q}(\beta)$, where $a_{Q P}=\left\langle T \psi_{P}, \varphi_{Q}\right\rangle$.

Remark 8.8. The definition of $T \in \mathbf{A D}_{p}^{\alpha q}(\beta)$ is independent of the choice of the pair $(\varphi, \psi)$.

Proof. Define $\mathcal{S}_{0}=\{f \in \mathcal{S}: 0 \notin \operatorname{supp} \hat{f}\}$. Observe that $\psi \in \mathcal{A}$ implies $\psi, \psi_{\nu}, \psi_{Q} \in$ $\mathcal{S}_{0}$ for $\nu \in \mathbb{Z}$ and $Q$ dyadic. Moreover, if $g \in \mathcal{S}_{0}$, then both

$$
g_{N}:=\sum_{\nu=-N}^{N} \sum_{k \in \mathbb{Z}^{n}} s_{Q_{\nu k}}(g) \psi_{Q_{\nu k}} \underset{N \rightarrow \infty}{\rightarrow} g
$$

and $\sum_{|k| \leq M} s_{Q_{\nu k}}(g) \psi_{Q_{\nu k}} \underset{M \rightarrow \infty}{\rightarrow} \sum_{k \in \mathbb{Z}^{n}} s_{Q_{\nu k}}(g) \psi_{Q_{\nu k}}$ in the $\mathcal{S}$-topology (see [13, Appendix]). Since $T$ is continuous from $\mathcal{S}$ into $\mathcal{S}^{\prime}$, we have $T g=\sum_{Q} s_{Q}(g) T \psi_{Q}$. Now, suppose $\left(\left\langle T \psi_{P}, \varphi_{Q}\right\rangle_{Q P}\right) \in \mathbf{a d}_{p}^{\alpha q}(\beta)$ for some fixed pair $(\varphi, \psi)$ of mutually admissible kernels. Take any other such pair $(\tilde{\varphi}, \tilde{\psi})$. Then $\tilde{\psi}_{P}=\sum_{L}\left\langle\tilde{\psi}_{P}, \varphi_{L}\right\rangle \psi_{L}$ and $\tilde{\varphi}_{Q}=\sum_{R}\left\langle\tilde{\varphi}_{Q}, \psi_{R}\right\rangle \varphi_{R}$, which gives

$$
\left\langle T \tilde{\psi}_{P}, \tilde{\varphi}_{Q}\right\rangle=\sum_{R, L}\left\langle\tilde{\psi}_{P}, \varphi_{L}\right\rangle\left\langle T \psi_{L}, \varphi_{R}\right\rangle \overline{\left\langle\tilde{\varphi}_{Q}, \psi_{R}\right\rangle}
$$


Since both $\left\{\psi_{R}\right\}_{R}$ and $\left\{\varphi_{L}\right\}_{L}$ constitute families of smooth molecules for $\dot{B}_{p}^{\alpha q}(W)$, by (18.2) the matrices $\left(\left\langle\tilde{\psi}_{P}, \varphi_{L}\right\rangle_{L P}\right),\left(\left\langle\tilde{\varphi}_{Q}, \psi_{R}\right\rangle_{Q R}\right) \in \mathbf{a d}_{p}^{\alpha q}(\beta)$. By Theorem 8.5 $\left(\left\langle T \tilde{\psi}_{P}, \tilde{\varphi}_{Q}\right\rangle_{Q P}\right) \in \mathbf{a d}_{p}^{\alpha q}(\beta)$.

A straightforward consequence of Theorem [1.10 is the following statement:

Corollary 8.9. Let $T \in \mathbf{A D}_{p}^{\alpha q}(\beta), \alpha \in \mathbb{R}, 1 \leq p<\infty, 0<q<\infty$. Then Textends to a bounded operator on $\dot{B}_{p}^{\alpha q}(W)$ if $W$ satisfies any of (A1)-(A3).

Proof. First, consider $\vec{f}$ with $(\vec{f})_{i} \in \mathcal{S}_{0}$. Let $(\varphi, \psi)$ be a pair of mutually admissible kernels. Denote $\vec{t}_{Q}=\sum_{P}\left\langle T \psi_{P}, \varphi_{Q}\right\rangle \vec{s}_{P}(\vec{f})$ and observe that $\left(\left\langle T \psi_{P}, \varphi_{Q}\right\rangle_{Q P}\right) \in$ $\operatorname{ad}_{p}^{\alpha q}(\beta)$. Using the $\varphi$-transform decomposition $\vec{f}=\sum_{P} \vec{s}_{P}(\vec{f}) \psi_{P}$ and taking $T$ inside the sum as in the previous remark, we get

$$
\begin{aligned}
\|T \vec{f}\|_{\dot{B}_{p}^{\alpha q}(W)} & =\left\|\sum_{P} \vec{s}_{P}(\vec{f}) T \psi_{P}\right\|_{\dot{B}_{p}^{\alpha q}(W)} \\
& =\left\|\sum_{Q}\left(\sum_{P}\left\langle T \psi_{P}, \varphi_{Q}\right\rangle \vec{s}_{P}(\vec{f})\right) \psi_{Q}\right\|_{\dot{B}_{p}^{\alpha q}(W)} \\
& =\left\|\sum_{Q} \vec{t}_{Q} \psi_{Q}\right\|_{\dot{B}_{p}^{\alpha q}(W)} \leq c\left\|\left\{\vec{t}_{Q}\right\}_{Q}\right\|_{\dot{b}_{p}^{\alpha q}(W)} \\
& \leq c\left\|\left\{\vec{s}_{Q}\right\}_{Q}\right\|_{\dot{b}_{p}^{\alpha q}(W)} \leq c\|\vec{f}\|_{\dot{B}_{p}^{\alpha q}(W)},
\end{aligned}
$$

by Corollary 5.6, Theorem 1.10 and Theorem 6.6 .

Note that $\mathcal{S}_{0}$ is dense in $\dot{B}_{p}^{\alpha q}(W)$ (since $\left.q<\infty\right)$ and $W$ satisfies any of (A1)(A3) (this follows from Corollary 5.6, Theorem 6.6 and the fact that the tail of a convergent series goes to zero, see [13, Appendix]). Thus, $T$ extends to all of $\dot{B}_{p}^{\alpha q}(W)$.

Note that if $q=\infty$, then $T$ extends to a bounded operator on the closure of $\mathcal{S}_{0}$ in $\dot{B}_{p}^{\alpha \infty}(W)$.

Remark 8.10. Let $\left\{m_{Q}\right\}_{Q}$ be a family of smooth molecules for $\dot{B}_{p}^{\alpha q}(W)$. Apply the $\varphi$-transform to $\sum_{P} \vec{s}_{P} m_{P}$ :

$$
\vec{t}_{Q}:=S_{\varphi}\left(\sum_{P} \vec{s}_{P} m_{P}\right)=\left\langle\sum_{P} \vec{s}_{P} m_{P}, \varphi_{Q}\right\rangle=\sum_{P}\left\langle m_{P}, \varphi_{Q}\right\rangle \vec{s}_{P} .
$$

Then $\left(\left\langle m_{P}, \varphi_{Q}\right\rangle_{Q P}\right)$ forms an almost diagonal matrix by (8.2), and therefore, by Theorem 1.10,

$$
\left\|\left\{\vec{t}_{Q}\right\}_{Q}\right\|_{\dot{b}_{p}^{\alpha q}(W)} \equiv\left\|S_{\varphi}\left(\sum_{P} \vec{s}_{P} m_{P}\right)\right\|_{\dot{b}_{p}^{\alpha q}(W)} \leq c\left\|\left\{\vec{s}_{P}\right\}_{P}\right\|_{\dot{b}_{p}^{\alpha q}(W)}
$$

if $W$ is doubling.

Corollary 8.11. Let $T, S \in \mathbf{A D}_{p}^{\alpha q}(\beta)$. Then $T \circ S \in \mathbf{A D}_{p}^{\alpha q}(\beta)$. 
Proof. Since $T, S \in \mathbf{A D}_{p}^{\alpha q}(\beta)$, it follows that $\left(t_{Q P}\right):=\left(\left\langle T \psi_{P}, \varphi_{Q}\right\rangle_{Q P}\right)$ is in $\operatorname{ad}_{p}^{\alpha q}(\beta)$, and so is $\left(s_{Q P}\right):=\left(\left\langle S \psi_{P}, \varphi_{Q}\right\rangle_{Q P}\right)$. Thus, for $Q, P$ dyadic we have $S \psi_{P}=\sum_{R}\left\langle S \psi_{P}, \varphi_{R}\right\rangle \psi_{R}$, and so

$$
\left\langle T \circ S \psi_{P}, \varphi_{Q}\right\rangle=\sum_{R}\left\langle S \psi_{P}, \varphi_{R}\right\rangle\left\langle T \psi_{R}, \varphi_{Q}\right\rangle=\sum_{R} t_{Q R} s_{R P} \in \operatorname{ad}_{p}^{\alpha q}(\beta),
$$

by Theorem 8.5 (composition of almost diagonal matrices).

\section{Calderón-Zygmund operators}

In this section we show that Calderón-Zygmund operators (CZOs) are bounded on $\dot{B}_{p}^{\alpha q}(W)$ for certain parameters $\alpha, p, q, \beta$. First we recall the definition of smooth atoms and the fact that a CZO maps smooth atoms into smooth molecules. Then we use a general criterion for boundedness of operators: if an operator $T$ maps smooth atoms into molecules, then its matrix $\left(\left\langle T \psi_{P}, \varphi_{Q}\right\rangle_{Q P}\right)$ forms an almost diagonal operator on $\dot{b}_{p}^{\alpha q}(W)$, and therefore, $T$ is bounded on $\dot{B}_{p}^{\alpha q}(W)$.

Definition 9.1. Let $N \in \mathbb{N} \cup\{0\}$. A function $a_{Q} \in \mathcal{D}\left(\mathbb{R}^{n}\right)$ is a smooth $N$-atom for $Q$ if

1. $\operatorname{supp} a_{Q} \subseteq 3 Q$,

2. $\int x^{\gamma} a_{Q}(x) d x=0$ for $|\gamma| \leq N$, and

3. $\left|D^{\gamma} a_{Q}(x)\right| \leq c_{\gamma} l(Q)^{-|\gamma|-n / 2}$ for all $|\gamma| \geq 0$.

Let $0<\delta \leq 1, M>0, N \in \mathbb{N} \cup\{0,-1\}, N_{0} \in \mathbb{N} \cup\{0\}$.

Lemma 9.2 (BOUNDEDNESS CRITERION). Suppose a continuous linear operator $T: \mathcal{S} \rightarrow \mathcal{S}^{\prime}$ maps any smooth $N_{0}$-atom into a fixed multiple of a smooth $(\delta, M, N)$ molecule for $\dot{B}_{p}^{\alpha q}(W), \alpha \in \mathbb{R}, 1 \leq p<\infty, 0<q \leq \infty$ with $\delta, M, N$ satisfying (M.i), (M.ii) and (M.iii). Suppose $W$ satisfies any of (A1)-(A3). Then $T \in \mathbf{A D}_{p}^{\alpha q}(\beta)$ and, if $q<\infty, T$ extends to a bounded operator on $\dot{B}_{p}^{\alpha q}(W)$.

Proof. By Corollary [8.9, it suffices to show that $\left(\left\langle T \psi_{P}, \varphi_{Q}\right\rangle_{Q P}\right) \in \operatorname{ad}_{p}^{\alpha q}(\beta)$ for some $\varphi, \psi \in \mathcal{A}$ satisfying (2.1). Observe that if $\psi \in \mathcal{A}$, then there exists $\theta \in \mathcal{S}$ with $\operatorname{supp} \theta \subseteq B_{1}(0), \int x^{\gamma} \theta(x) d x=0$, if $|\gamma| \leq N_{0}$, and $\sum_{\nu \in \mathbb{Z}} \hat{\theta}\left(2^{-\nu} \xi\right) \hat{\varphi}\left(2^{-\nu} \xi\right)=1$ for $\xi \neq 0$ (3], Lemma 5.12). Using $\psi_{P}=\sum_{\nu \in \mathbb{Z}} \theta_{\nu} * \varphi_{\nu} * \psi_{P}$ as in the atomic decomposition theorem ([3], Thm. 5.11), we have

$$
\psi_{P}(x)=\sum_{Q} t_{Q P} a_{Q}^{(P)}(x)
$$

with $t_{Q P}=|Q|^{1 / 2} \sup _{y \in Q}\left|\left(\varphi_{\nu} * \psi_{P}\right)(y)\right|$ for $l(Q)=2^{-\nu}$, and each $a_{Q}^{(P)}$ is an $N_{0}$-atom defined by

$$
a_{Q}^{(P)}(x)=\frac{1}{t_{Q P}} \int_{Q} \theta_{\nu}(x-y)\left(\varphi_{\nu} * \psi_{P}\right)(y) d y \quad \text { if } t_{Q P} \neq 0
$$


and $a_{Q}^{(P)}=0$ if $t_{Q P}=0$. Using (5.2)-(5.3) (valid because $\left\{\psi_{P}\right\}_{P}$ is a family of molecules for $\dot{B}_{p}^{\alpha q}(W)$ ), we get

$$
\left|\left(\varphi_{\nu} * \psi_{P}\right)(y)\right| \leq c|P|^{-1 / 2} \min \left(\left[\frac{l(Q)}{l(P)}\right]^{\tau},\left[\frac{l(P)}{l(Q)}\right]^{\sigma}\right)\left(1+\frac{\left|y-x_{P}\right|}{\max (l(Q), l(P))}\right)^{-M}
$$

for some $\tau>\alpha$ and $\sigma>J-\alpha$. In fact, $\varphi_{\nu} * \psi_{P}=0$ if $|\mu-\nu|>1\left(2^{-\mu}=l(P)\right)$, since $\varphi, \psi \in \mathcal{A}$, but all we require is the previous estimate. Since $y \in Q, y$ can be replaced by $x_{Q}$ in the last expression by Lemma 8.4, and so

$$
\left|t_{Q P}\right| \leq c\left(\frac{|Q|}{|P|}\right)^{1 / 2} \min \left(\left[\frac{l(Q)}{l(P)}\right]^{\tau},\left[\frac{l(P)}{l(Q)}\right]^{\sigma}\right)\left(1+\frac{\left|x_{Q}-x_{P}\right|}{\max (l(Q), l(P))}\right)^{-M},
$$

which is exactly (8.1). Thus $\left(t_{Q P}\right) \in \mathbf{a d}_{p}^{\alpha q}(\beta)$. Using (9.1), we obtain

$$
\left\langle T \psi_{P}, \varphi_{Q}\right\rangle=\left\langle\sum_{R} t_{R P} T a_{R}^{(P)}, \varphi_{Q}\right\rangle=\sum_{R} t_{R P}\left\langle T a_{R}^{(P)}, \varphi_{Q}\right\rangle .
$$

Since $T$ maps any $N_{0}$-atom $a_{R}^{(P)}$ into a fixed multiple of a smooth $(\delta, M, N)$-molecule $m_{R}: T a_{R}^{(P)}=c m_{R}$ and $c$ depends neither on $R$ nor on $Q$, we get

$$
\left\langle T a_{R}^{(P)}, \varphi_{Q}\right\rangle=c\left\langle m_{R}, \varphi_{Q}\right\rangle=: c \tilde{t}_{Q R}
$$

and by 8.2 , since $m_{R}$ is a smooth $(\delta, M, N)$-molecule for $\dot{B}_{p}^{\alpha q}(W),\left(\tilde{t}_{Q R}\right) \in$ $\operatorname{ad}_{p}^{\alpha q}(\beta)$. Hence,

$$
\left(\left\langle T \psi_{P}, \varphi_{Q}\right\rangle_{Q P}\right)=\left(c \sum_{R} \tilde{t}_{Q R} t_{R P}\right) \in \mathbf{a d}_{p}^{\alpha q}(\beta),
$$

since the composition of two almost diagonal operators is again almost diagonal by Theorem 8.11.

Let $T$ be a continuous linear operator from $\mathcal{S}\left(\mathbb{R}^{n}\right)$ to $\mathcal{S}^{\prime}\left(\mathbb{R}^{n}\right)$, and let $K=K(x, y)$ be its distributional kernel defined on $\mathbb{R}^{2 n} \backslash \Delta$, where $\Delta=\left\{(x, y) \in \mathbb{R}^{n} \times \mathbb{R}^{n}: x=\right.$ $y\}$ (for definitions refer to [3], Chapter 8). Then $T \in C Z O(\epsilon), 0<\epsilon \leq 1$, if $K$ has the following properties:

$$
\begin{aligned}
& \text { (I) }|K(x, y)| \leq \frac{c}{|x-y|^{n}}, \\
& \left(\mathrm{II}_{\epsilon}\right)\left|K(x, y)-K\left(x^{\prime}, y\right)\right|+\left|K(y, x)-K\left(y, x^{\prime}\right)\right| \leq c \frac{\left|x-x^{\prime}\right|^{\epsilon}}{|x-y|^{n+\epsilon}} \text { if } 2\left|x-x^{\prime}\right| \leq|x-y| .
\end{aligned}
$$

To show that a CZO maps atoms into molecules we start with the following result from [3]:

Theorem 9.3 ([3], Thm. 8.13). Let $0<\epsilon \leq 1$ and $0<\alpha<1$. If $T \in C Z O(\epsilon) \cap$ $W B P$ and $T 1=0$, then $T$ maps any smooth 0 -atom $a_{Q}$ into a fixed multiple of a smooth $(\epsilon, n+\epsilon,-1)$-molecule $m_{Q}$.

Thus, if $a_{Q}$ is a smooth 0-atom for $Q$, then $T a_{Q}=c m_{Q}$, where $m_{Q}$ satisfies
1. $\left|m_{Q}(x)\right| \leq|Q|^{-1 / 2}\left(1+\frac{\left|x-x_{Q}\right|}{l(Q)}\right)^{-(n+\epsilon)}$,
2. $\left|m_{Q}(x)-m_{Q}(y)\right| \leq|Q|^{-\frac{1}{2}}\left(\frac{|x-y|}{l(Q)}\right)^{\epsilon} \sup _{|z| \leq|x-y|}\left(1+\frac{\left|x-z-x_{Q}\right|}{l(Q)}\right)^{-(n+\epsilon)}$, 
and $c$ is uniform for all $Q$. Moreover, an $(\epsilon, n+\epsilon,-1)$-molecule is a smooth molecule for $\dot{B}_{p}^{\alpha q}(W)$ (see Section 5) if $1<p<\infty, 0<q \leq \infty, 0<\alpha<\epsilon$ and $\beta<n+p \alpha$ :

(i) $\delta=\epsilon$ and $0<\alpha<\epsilon \leq 1$,

(ii) $J=\frac{n}{p^{\prime}}+\frac{\beta}{p}<n+\alpha<n+\epsilon=M$,

(iii) $J-n-\alpha=\frac{\beta-n}{p}-\alpha<0 \Longrightarrow N=\max ([J-n-\alpha],-1)=-1$.

The next theorem follows by combining the two statements mentioned above and gives the boundedness of certain Calderón-Zygmund operators on $\dot{B}_{p}^{\alpha q}(W)$ with some restriction on the weight $W$ :

Theorem 9.4. Suppose $0<\epsilon \leq 1,0<\alpha<\epsilon, 1 \leq p<\infty, 0<q<\infty$, and $W$ satisfies any of (A1)-(A3). Assume $\beta<n+p \alpha$. If $T \in C Z O(\epsilon) \cap W B P$ and $T 1=0$, then $T$ extends to a bounded operator on $\dot{B}_{p}^{\alpha q}(W)$.

Remark 9.5. If also $T^{*} 1=0$ in Theorem 9.3 , then $\alpha=0$ can be included into the range, since $\int T a(x) d x=\langle T a, 1\rangle=\left\langle a, T^{*} 1\right\rangle=0$ and so $T$ maps any smooth 0 -atom into a smooth $(\epsilon, n+\epsilon, 0)$-molecule (see also [3], Cor. 8.21).

Corollary 9.6. Let $1 \leq p<\infty, 0<q<\infty, 0<\epsilon \leq 1$ and $0 \leq \alpha<\epsilon$. Assume $\beta<n+p \epsilon$. If $T \in C Z O(\epsilon) \cap W B P$ and $T 1=T^{*} 1=0$, then $T$ extends to a bounded operator on $\dot{B}_{p}^{\alpha q}(W)$, in particular, for $\alpha=0$.

Proof. Since $N=0$, the bound on $\beta$ from the previous theorem can be relaxed to $\beta<n+p \epsilon$.

Remark 9.7. The condition $T^{*}\left(y^{\gamma}\right)=0$ for $|\gamma| \leq N, N \geq 1$, produces more vanishing moments of a molecule $T a$; so it is not difficult to satisfy (M.iii). But (M.ii) $M=n+\epsilon>J=n+\frac{\beta-n}{p} \Longleftrightarrow \beta<n+p \epsilon$ creates a major restriction on the doubling exponent of $W$. Note that in this case, we get that $T$ maps any smooth 0 -atom into a smooth $(\epsilon, n+\epsilon, N)$-molecule, but this molecule is not a smooth molecule for $\dot{B}_{p}^{\alpha q}(W)$. From now on $N \geq 0$, since the case $N=-1$ is completely covered by Theorem 9.4 .

Now we want to show that the restriction on the weight $W$ (to be more precise, the restriction on the doubling exponent $\beta$ ) can be removed in some cases by requiring more smoothnes than $\left(\mathrm{II}_{\epsilon}\right)$ on the kernel $K$.

We say that $T \in C Z O(N+\epsilon), N \in \mathbb{N} \cup\{0\}, 0<\epsilon \leq 1$, if $T$ is a continuous linear operator from $\mathcal{S}\left(\mathbb{R}^{n}\right)$ to $\mathcal{S}^{\prime}\left(\mathbb{R}^{n}\right)$ and $K$, its distributional kernel defined on $\mathbb{R}^{2 n} \backslash \Delta$, has the following properties:

(I) $|K(x, y)| \leq \frac{c}{|x-y|^{n}}$,

$\left(\mathrm{II}_{N}\right)\left|D_{(2)}^{\gamma} K(x, y)\right| \leq \frac{c}{|x-y|^{n+|\gamma|}}$, for $|\gamma| \leq N$, and

$\left(\mathrm{II}_{N+\epsilon}\right)$ The inequality

$$
\begin{aligned}
& \left|D_{(2)}^{\gamma} K(x, y)-D_{(2)}^{\gamma} K\left(x^{\prime}, y\right)\right|+\left|D_{(2)}^{\gamma} K(y, x)-D_{(2)}^{\gamma} K\left(y, x^{\prime}\right)\right| \\
& \quad \leq c \frac{\left|x-x^{\prime}\right|^{\epsilon}}{|x-y|^{n+|\gamma|+\epsilon}}
\end{aligned}
$$

holds for $2\left|x-x^{\prime}\right| \leq|x-y|$ and $|\gamma|=N$,

where the subscript 2 in $D_{(2)}^{\gamma}$ refers to differentiation with respect to the second argument of $K(x, y)$.

Note that $C Z O(\epsilon) \supseteq C Z O(N+\epsilon)$, for $N \geq 0$. 
Theorem 9.8. Let $0 \leq \alpha<1,0<\epsilon \leq 1, N_{0} \in \mathbb{N} \cup\{0\}$. Suppose $T \in C Z O\left(N_{0}+\right.$ $\epsilon) \cap W B P, T 1=0$ and $T^{*}\left(y^{\gamma}\right)=0$ for $|\gamma| \leq N_{0}$. Then $T$ maps any $N_{0}$-atom $a_{Q}$ into a fixed multiple of a smooth $\left(\epsilon, N_{0}+n+\epsilon, N_{0}\right)$-molecule.

More precisely, we will show that $T a_{Q}=c m_{Q}$ with $c$ independent of $Q$ and

(i) $\int x^{\gamma} T a_{Q}(x) d x=0$, for $|\gamma| \leq N_{0}$,

(ii) $\left|T a_{Q}(x)\right| \leq c|Q|^{-1 / 2}\left(1+\frac{\left|x-x_{Q}\right|}{l(Q)}\right)^{-\left(N_{0}+n+\epsilon\right)}$,

(iii) $\left|T a_{Q}(x)-T a_{Q}(y)\right| \leq c|Q|^{-\frac{1}{2}}\left[\frac{|x-y|}{l(Q)}\right]^{\epsilon}$

$$
\times \sup _{|z| \leq|x-y|}\left(1+\frac{\left|z-\left(x-x_{Q}\right)\right|}{l(Q)}\right)^{-\left(N_{0}+n+\epsilon\right)} .
$$

Before we start the proof, we quote the following estimate:

Lemma 9.9 ([10]). Let $T: \mathcal{D} \rightarrow \mathcal{D}^{\prime}$ be a continuous linear operator with $T \in$ $C Z O(\epsilon) \cap W B P, 0<\epsilon \leq 1$, and $T 1=0$. Then $T$ maps $\mathcal{D}$ into $L^{\infty}$, and there exists a constant $c$ such that for any fixed $z \in \mathbb{R}^{n}, t>0, \varphi \in \mathcal{D}$ with $\operatorname{supp} \varphi \in B_{t}(z)$,

$$
\|T \varphi\|_{L^{\infty}} \leq c\left(\|\varphi\|_{L^{\infty}}+t\|\nabla \varphi\|_{L^{\infty}}\right) .
$$

Proof of Theorem 9.8. For simplicity, we give the proofs of (i), (ii) and (iii) for $Q=Q_{00}$. The same methods apply to the general cube because of the dilationtranslation nature of the estimates. Thus, consider the unit atom $a=a_{Q_{00}}$ with $x_{Q_{00}}=0$ and $l\left(Q_{00}\right)=1$. First, property (i) immediately follows from the fact that $T^{*}\left(y^{\gamma}\right)=0$ for $|\gamma| \leq N_{0}$. To get (ii) we consider two cases: $|x| \leq 6 \sqrt{n}$ and $|x|>6 \sqrt{n}$. For $|x| \leq 6 \sqrt{n}$, use Lemma 9.9 to obtain

$$
|T a(x)| \leq\|T a\|_{L^{\infty}} \leq c\left(\|a\|_{L^{\infty}}+\|\nabla a\|_{L^{\infty}}\right) \leq c .
$$

If $|x|>6 \sqrt{n}$, we get

$$
\begin{aligned}
|T a(x)| & =\left|\int K(x, y) a(y) d y\right| \\
& =\left|\int_{3 Q_{00}}\left[K(x, y)-\sum_{|\gamma| \leq N_{0}} \frac{D_{(y)}^{\gamma} K(x, 0)}{\gamma !} y^{\gamma}\right] a(y) d y\right|,
\end{aligned}
$$

since $a_{Q}$ is an $N_{0}$-atom, and thus, has $N_{0}$ vanishing moments $\int y^{\gamma} a_{Q}(y) d y=0$ for $|\gamma| \leq N_{0}$. Then (9.3) is bounded by

$$
\int_{3 Q_{00}} \sum_{|\gamma|=N_{0}}\left|\left[D_{(y)}^{\gamma} K(x, \theta(y))-D_{(y)}^{\gamma} K(x, 0)\right] \frac{y^{\gamma}}{\gamma !}\right||a(y)| d y .
$$

Note that if $y \in \operatorname{supp} a$, then $2|\theta(y)| \leq 2|y| \leq 2 \cdot 3 \sqrt{n}<|x|$, and, using the property $\left(\mathrm{II}_{N+\epsilon}\right)$ of the kernel $K$ to estimate the difference, we get

$$
\sum_{|\gamma|=N_{0}}\left|D_{(y)}^{\gamma} K(x, \theta(y))-D_{(y)}^{\gamma} K(x, 0)\right| \leq c \frac{|\theta(y)|^{\epsilon}}{|x|^{n+|\gamma|+\epsilon}} \leq c \frac{|y|^{\epsilon}}{|x|^{n+N_{0}+\epsilon}} .
$$

Thus,

$$
|T a(x)| \leq \frac{c_{n, N_{0}}}{|x|^{n+N_{0}+\epsilon}} \int_{3 Q_{00}}|y|^{N_{0}+\epsilon}|a(y)| d y \leq \frac{c}{|x|^{n+N_{0}+\epsilon}} .
$$


In order to show (iii), we prove that

$$
\left|T a(x)-T a\left(x^{\prime}\right)\right| \leq c\left|x-x^{\prime}\right|^{\epsilon}\left(\frac{1}{(1+|x|)^{n+N_{0}+\epsilon}}+\frac{1}{\left(1+\left|x^{\prime}\right|\right)^{n+N_{0}+\epsilon}}\right) .
$$

In the case $\left|x-x^{\prime}\right| \geq 1$, the estimate (9.4) follows trivially from (ii) and the triangle inequality. For $\left|x-x^{\prime}\right|<1$ and $|x|>10 \sqrt{n}$, we can use vanishing moments of $a(x)$ and the integral form of the remainder to get

$$
\begin{aligned}
& \left|T a(x)-T a\left(x^{\prime}\right)\right|=\left|\int\left(K(x, y)-K\left(x^{\prime}, y\right)\right) a(y) d y\right|=\mid \int_{3 Q_{00}}[K(x, y) \\
& \left.-\sum_{|\gamma| \leq N_{0}-1} \frac{D_{(y)}^{\gamma} K(x, 0)}{\gamma !} y^{\gamma}-K\left(x^{\prime}, y\right)+\sum_{|\gamma| \leq N_{0}-1} \frac{D_{(y)}^{\gamma} K\left(x^{\prime}, 0\right)}{\gamma !} y^{\gamma}\right] a(y) d y \mid \\
\leq & \int_{3 Q_{00}} \int_{0}^{1} \frac{(1-s)^{N_{0}-1}}{\left(N_{0}-1\right) !} \sum_{|\gamma|=N_{0}}\left|D_{(y)}^{\gamma} K(x, s y)-D_{(y)}^{\gamma} K\left(x^{\prime}, s y\right)\right| \frac{|y|^{\gamma}}{\gamma !}|a(y)| d s d y .
\end{aligned}
$$

If $|x| \geq 10 \sqrt{n}$ and $y \in \operatorname{supp} a$, then $|x-s y| \geq|x|-s|y| \geq 10 \sqrt{n}-3 \sqrt{n} \geq 2\left|x-x^{\prime}\right|$ and also $|x-s y| \geq|x|-s|y| \geq|x|-3 \sqrt{n} \geq|x|-\frac{|x|}{2} \geq \frac{|x|}{2}$. By $\left(\operatorname{II}_{N+\epsilon}\right)$, the last integral is bounded by

$$
c \int_{3 Q_{00}} \int_{0}^{1} \frac{\left|x-x^{\prime}\right|^{\epsilon}}{|x-s y|^{n+N_{0}+\epsilon}}|y|^{N_{0}} d s d y \leq c \frac{\left|x-x^{\prime}\right|^{\epsilon}}{|x|^{n+N_{0}+\epsilon}} .
$$

In case $\left|x-x^{\prime}\right|<1$ and $|x| \leq 10 \sqrt{n}$, an exact repetition of the argument on p. 85 of [3] or part (c) on p. 62 of [6] shows that

$$
\left|T a(x)-T a\left(x^{\prime}\right)\right| \leq c\left|x-x^{\prime}\right|^{\epsilon}
$$

by using the decay property (I) and the Lipschitz condition $\left(\mathrm{II}_{0+\epsilon}\right)$ of the kernel $K$, which holds for any $C Z O\left(N_{0}+\epsilon\right), N_{0} \geq 0$. This completes the proof.

Corollary 9.10. Let $1 \leq p<\infty, 0<q<\infty$, and let $W$ satisfy any of (A1)(A3). Suppose $0 \leq \alpha \leq \frac{\beta-n}{p}-\left[\frac{\beta-n}{p}\right]$, where $\beta$ is the doubling exponent of $W$. Let $N=\left[\frac{\beta-n}{p}-\alpha\right]$ and $\frac{\beta-n}{p}-\left[\frac{\beta-n}{p}\right]<\epsilon \leq 1$. If $T \in C Z O(N+\epsilon) \cap W B P, T 1=0$ and $T^{*}\left(y^{\gamma}\right)=0$ for $|\gamma| \leq N$, then $T$ extends to a bounded operator on $\dot{B}_{p}^{\alpha q}(W)$.

Proof. By the previous theorem, $T$ maps any smooth $N$-atom into a smooth $(\epsilon, N+$ $n+\epsilon, N)$-molecule. This molecule is a smooth molecule for $\dot{B}_{p}^{\alpha q}(W)$ if (i) $\alpha<\epsilon \leq 1$, (ii) $M=N+n+\epsilon>J=n+\frac{\beta-n}{p} \Longleftrightarrow\left[\frac{\beta-n}{p}-\alpha\right]=\left[\frac{\beta-n}{p}\right]>\frac{\beta-n}{p}-\epsilon$, and (iii) $N=\max ([J-n-\alpha],-1)=\left[\frac{\beta-n}{p}-\alpha\right]$, which are all true. By the boundedness criterion (Lemma 9.2), $T$ is bounded on $\dot{B}_{p}^{\alpha q}(W)$.

Corollary 9.11. Let $1 \leq p<\infty, 0<q<\infty$, and let $W$ satisfy any of (A1)-(A3). Suppose $0 \leq \frac{\beta-n}{p}-\left[\frac{\beta-n}{p}\right]<\alpha<1$, where $\beta$ is the doubling exponent of $W$. Let $N=\left[\frac{\beta-n}{p}-\alpha\right]$ and $\alpha<\epsilon \leq 1$. If $T \in C Z O(N+1+\epsilon) \cap W B P, T 1=0$ and $T^{*}\left(y^{\gamma}\right)=0$ for $|\gamma| \leq N+1$, then $T$ is bounded on $\dot{B}_{p}^{\alpha q}(W)$. 
Proof. By Theorem 9.8, $T$ maps any smooth $(N+1)$-atom into a smooth $(\epsilon, N+$ $1+n+\epsilon, N+1)$-molecule, which is also a smooth $(\epsilon, N+1+n+\epsilon, N)$-molecule. This one, in its turn, is a smooth molecule for $\dot{B}_{p}^{\alpha q}(W)$, since (i) $\alpha<\epsilon \leq 1$, (ii) $M=N+1+n+\epsilon>J=n+\frac{\beta-n}{p} \Longleftrightarrow\left[\frac{\beta-n}{p}-\alpha\right]+1>\frac{\beta-n}{p}-\epsilon$, and (iii) $N=\max ([J-n-\alpha],-1)=\left[\frac{\beta-n}{p}-\alpha\right]$. By the boundedness criterion (Lemma 9.2), $T$ extends to a bounded operator on $\dot{B}_{p}^{\alpha q}(W)$.

Remark 9.12. Note that the condition $T^{*}\left(y^{\gamma}\right)=0,|\gamma| \leq N$, can be very restrictive; for example, the Hilbert transform does not satisfy this condition for $|\gamma|>0$. On the other hand, we have considered a general class of CZOs, not necessarily of convolution type. Utilizing the convolution structure will let us drop the above condition.

Let $N \in \mathbb{N} \cup\{0\}$. Let $T$ be a convolution operator, i.e., the kernel $K(x, y)=$ $K(x-y)$ is defined on $\mathbb{R}^{n} \backslash\{0\}$ and satisfies

$$
\begin{aligned}
& \text { (C.1) }|K(x)| \leq \frac{c}{|x|^{n}}, \\
& \text { (C.2) }\left|D^{\gamma} K(x)\right| \leq \frac{c}{|x|^{n+|\gamma|}} \text {, for }|\gamma| \leq N+1, \\
& \text { (C.3) } \int_{R_{1}<|x|<R_{2}} K(x) d x=0 \text {, for all } 0<R_{1}<R_{2}<\infty .
\end{aligned}
$$

Remark 9.13. We replace $\left(\mathrm{II}_{N}\right)$ and $\left(\mathrm{II}_{N+\epsilon}\right)$ of the general $\mathrm{CZO}$ kernel with the slightly stronger smoothness condition (C.2) to make the proof below more concise. The reader can check that conditions corresponding to $\left(\mathrm{II}_{N}\right)$ and $\left(\mathrm{II}_{N+\epsilon}\right)$ in the convolution case would suffice for the statements below.

Now we obtain an analog of Theorem 9.8 saying that $T$ maps smooth atoms into smooth molecules, and then we show the boundedness of $T$.

Theorem 9.14. Let $0 \leq \alpha<1,0<\epsilon \leq 1, N \in \mathbb{N} \cup\{0\}$. Let $T$ be a convolution operator with a kernel $K$ satisfying (C.1)-(C.3). Then $T$ maps any smooth $N$-atom $a_{Q}$ into a fixed multiple of a smooth $(\epsilon, N+1+n, N)$-molecule.

More precisely, we will show that

$$
\begin{aligned}
& \text { (i) } \int x^{\gamma} T a_{Q}(x) d x=0 \text { for }|\gamma| \leq N \text {, and } \\
& \text { (ii) }\left|D^{\gamma} T a_{Q}(x)\right| \leq c|Q|^{-1 / 2-|\gamma| / n}\left(1+\frac{\left|x-x_{Q}\right|}{l(Q)}\right)^{-(N+n+1)} \text { for }|\gamma|=0,1 .
\end{aligned}
$$

By the Mean Value Theorem, (ii) with $|\gamma|=1$ implies the Lipschitz condition (M4) for $|\gamma|=0$.

Proof. To obtain (ii) we first consider $x \notin 10 \sqrt{n} Q$. Then

$$
\left|T a_{Q}(x)\right|,\left|\nabla T a_{Q}(x)\right|=\left|\sum_{\left|\gamma_{0}\right|=0 \text { or } 1} \int_{3 Q} D^{\gamma_{0}} K(x-y) a_{Q}(y) d y\right|
$$




$$
\begin{aligned}
=\mid \sum_{\left|\gamma_{0}\right|=0 \text { or } 1} & \int_{3 Q}\left[D^{\gamma_{0}} K(x-y)\right. \\
& \left.-\sum_{|\gamma| \leq N-\left|\gamma_{0}\right|} \frac{D^{\gamma} D^{\gamma_{0}} K\left(x-x_{Q}\right)}{\gamma !}\left(x_{Q}-y\right)^{\gamma}\right] a_{Q}(y) d y \mid,
\end{aligned}
$$

since $a_{Q}$ is an $N$-atom, and thus, has $N$ vanishing moments $\int y^{\gamma} a_{Q}(y) d y=0$ for $|\gamma| \leq N$. Then (9.5) is bounded by

$$
\begin{array}{r}
\sum_{\left|\gamma_{0}\right|=0 \text { or } 1} \int_{3 Q} \sum_{|\gamma|=N+1-\left|\gamma_{0}\right|} \frac{\left|D^{\gamma+\gamma_{0}} K\left(x-x_{Q}+\theta\left(y-x_{Q}\right)\right)\right|}{\gamma !} \\
\times\left|x_{Q}-y\right|^{N-\left|\gamma_{0}\right|+1}\left|a_{Q}(y)\right| d y,
\end{array}
$$

for some $0 \leq \theta \leq 1$. Since $x \notin 10 \sqrt{n} Q$ and $y \in 3 Q,\left|y-x_{Q}\right| \leq 2 \sqrt{n} l(Q) \leq \frac{1}{2}\left|x-x_{Q}\right|$. Using property (C.2) of the kernel $K$, we get

$$
\left|D^{\gamma} K\left(x-x_{Q}+\theta\left(y-x_{Q}\right)\right)\right| \leq \frac{c}{\left|x-x_{Q}+\theta\left(y-x_{Q}\right)\right|^{n+|\gamma|}} \approx \frac{c}{\left|x-x_{Q}\right|^{n+|\gamma|}} .
$$

So,

$$
\begin{aligned}
& \left|T a_{Q}(x)\right|,\left|\nabla T a_{Q}(x)\right| \leq \frac{c_{n, N}}{\left|x-x_{Q}\right|^{n+N+1}} \int_{3 Q}\left|x_{Q}-y\right|^{N-\left|\gamma_{0}\right|+1}\left|a_{Q}(y)\right| d y \\
& \leq c \frac{[l(Q)]^{N-\left|\gamma_{0}\right|+1}}{\left|x-x_{Q}\right|^{n+N+1}}|Q|^{-1 / 2}|Q|=c|Q|^{-1 / 2-\left|\gamma_{0}\right| / n}\left[\frac{l(Q)}{\left|x-x_{Q}\right|}\right]^{n+N+1},
\end{aligned}
$$

by the properties of $a_{Q}$.

If $x \in 10 \sqrt{n} Q$ and $y \in 3 Q$, then $|x-y| \leq 13 n l(Q)$; so by the cancellation property (C.3) of $K$ (using $\left.D^{\gamma}\left(K * a_{Q}\right)=K *\left(D^{\gamma} a_{Q}\right)\right)$, we obtain

$$
\begin{aligned}
& \left|T a_{Q}(x)\right|,\left|\nabla T a_{Q}(x)\right| \leq \sum_{\left|\gamma_{0}\right|=0 \text { or } 1}\left|\int_{3 Q} K(x-y) D^{\gamma_{0}} a_{Q}(y) d y\right| \\
& \quad=\sum_{\left|\gamma_{0}\right|=0 \text { or } 1}\left|\int_{3 Q} K(x-y)\left[D^{\gamma_{0}} a_{Q}(y)-D^{\gamma_{0}} a_{Q}(x)\right] d y\right| \\
& \quad \leq c \int_{|y-x| \leq 13 n l(Q)} \frac{1}{|x-y|^{n}}|Q|^{-1 / 2-\left|\gamma_{0}\right| / n-1 / n}|x-y| d y \\
& \quad \leq c|Q|^{-1 / 2-\left|\gamma_{0}\right| / n-1 / n} \int_{0}^{13 n l(Q)} r^{-n} r r^{n-1} d r=c|Q|^{-1 / 2-\left|\gamma_{0}\right| / n} .
\end{aligned}
$$

This concludes the proof of (ii).

Property (i) comes from the fact that $T$ is a convolution operator and $a_{Q}$ has vanishing moments up to order $N$. Property (ii) guarantees the absolute convergence of the integral in (i).

Corollary 9.15. Convolution operators with kernels satisfying (C.1)-(C.3) are bounded on $\dot{B}_{p}^{\alpha q}(W)$ if $W$ satisfies any of (A1)-(A3) and $0 \leq \alpha<\epsilon \leq 1,0<q<\infty$, $1 \leq p<\infty$. In particular, the Hilbert transform $\mathbb{H}(n=1)$ is bounded on $\dot{B}_{p}^{\alpha q}(W)$ and the Riesz transforms $\mathcal{R}_{j}, j=1, \ldots, n$ ( $\left.n \geq 2\right)$, are bounded on $\dot{B}_{p}^{\alpha q}(W)$. 
Proof. This is an immediate consequence of Theorem 9.14 and Lemma 9.2 choose $N=\left[\frac{\beta-n}{p}-\alpha\right]$ in Theorem 9.14 then $T$ maps any smooth $N$-atom into a smooth $(\epsilon, N+1+n, N)$-molecule, which is either a smooth $(\epsilon, N+1+n, N)$-molecule for $\dot{B}_{p}^{\alpha q}(W)$, if $\alpha \leq \frac{\beta-n}{p}-\left[\frac{\beta-n}{p}\right]$, or an $(\epsilon, N+1+n, N-1)$-molecule for $\dot{B}_{p}^{\alpha q}(W)$, if $1>\alpha>\frac{\beta-n}{p}-\left[\frac{\beta-n}{p}\right]$. Note that both Hilbert and Riesz transforms are convolution type operators with kernels satisfying (C.1)-(C.3).

\section{WAVELETS}

Consider a pair $(\varphi, \psi)$ from $\mathcal{A}$ with the mutual property 2.1 . Then the family $\left\{\varphi_{Q}, \psi_{Q}\right\}$ behaves similarly to an orthonormal system because of the property

$$
f=\sum_{Q}\left\langle f, \varphi_{Q}\right\rangle \psi_{Q}=\sum_{Q} s_{Q} \psi_{Q} \text { for all } f \in \mathcal{S}^{\prime} / \mathcal{P}
$$

However, this system does not constitute an orthonormal basis. This can be achieved by the Meyer and Lemarié construction of a wavelet basis with the generating function $\theta \in \mathcal{S}$ (see [9] and [10]):

Theorem 10.1. There exist real-valued functions $\theta^{(i)} \in \mathcal{S}\left(\mathbb{R}^{n}\right), i=1, \ldots, 2^{n}-1$, such that the collection $\left\{\theta_{\nu k}^{(i)}\right\}=\left\{2^{\nu n / 2} \theta^{(i)}\left(2^{\nu} x-k\right)\right\}$ is an orthonormal basis for $L^{2}\left(\mathbb{R}^{n}\right)$. The functions $\theta^{(i)}$ satisfy

$$
\operatorname{supp} \hat{\theta}^{(i)} \subseteq\left\{\left[-\frac{8}{3} \pi, \frac{8}{3} \pi\right]^{n} \backslash\left[-\frac{2}{3} \pi, \frac{2}{3} \pi\right]^{n}\right\}
$$

and, hence,

$$
\int_{\mathbb{R}} x^{\gamma} \theta(x) d x=0 \text { for all multi-indices } \gamma
$$

Thus, we have $f=\sum_{i=1}^{2^{n}-1} \sum_{Q}\left\langle f, \theta_{Q}^{(i)}\right\rangle \theta_{Q}^{(i)}$ for all $f \in L^{2}\left(\mathbb{R}^{n}\right)$. This identity extends to all $f \in \mathcal{S}^{\prime} / \mathcal{P}\left(\mathbb{R}^{n}\right)$.

Theorem 10.2. Let $\alpha \in \mathbb{R}, 0<q \leq \infty, 1 \leq p<\infty$, and let $W$ satisfy any of (A1)-(A3). Let $\theta^{(i)}, i=1, \ldots, 2^{n}-1$, be generating wavelet functions as in Theorem 10.1. Then

$$
\|\vec{f}\|_{\dot{B}_{p}^{\alpha q}(W)} \approx \sum_{i=1}^{2^{n}-1}\left\|\left\{\left\langle\vec{f}, \theta_{Q}^{(i)}\right\rangle\right\}_{Q}\right\|_{\dot{b}_{p}^{\alpha q}(W)}
$$

Proof. Assume $i=1, \ldots, 2^{n}-1$. Since $\left\{\theta_{Q}^{(i)}\right\}_{Q, i}$ is a family of smooth molecules for $\dot{B}_{p}^{\alpha q}(W)$, the inequality

$$
\|\vec{f}\|_{\dot{B}_{p}^{\alpha q}(W)} \equiv\left\|\sum_{Q, i}\left\langle\vec{f}, \theta_{Q}^{(i)}\right\rangle \theta_{Q}^{(i)}\right\|_{\dot{B}_{p}^{\alpha q}(W)} \leq c \sum_{i}\left\|\left\{\left\langle\vec{f}, \theta_{Q}^{(i)}\right\rangle\right\}_{Q}\right\|_{\dot{b}_{p}^{\alpha q}(W)}
$$

follows immediately from Theorem [5.2. Therefore, we need to focus only on the opposite direction. 
Let $\varphi \in \mathcal{A}$ be such that $\sum_{\nu \in \mathbb{Z}}\left|\hat{\varphi}\left(2^{\nu} \xi\right)\right|^{2}=1$ for $\xi \neq 0$. Let $\vec{s}_{Q}=\left\langle\vec{f}, \varphi_{Q}\right\rangle$. Applying the boundedness of the $\varphi$-transform (Theorem 6.6), we obtain

$$
\left\|\left\{\vec{s}_{Q}\right\}_{Q}\right\|_{\dot{b}_{p}^{\alpha q}(W)} \leq c\|\vec{f}\|_{\dot{B}_{p}^{\alpha q}(W)} .
$$

Now for each $i$ and $Q$, define $\vec{t}_{Q}^{(i)}=\left\langle\vec{f}, \theta_{Q}^{(i)}\right\rangle$. Since $\theta_{Q}^{(i)} \in \mathcal{S}$, by the $\varphi$-transform decomposition with $\psi=\varphi$, we have $\theta_{Q}^{(i)}=\sum_{P}\left\langle\theta_{Q}^{(i)}, \varphi_{P}\right\rangle \varphi_{P}$, which gives

$$
\vec{t}_{Q}^{(i)}=\sum_{P} \overline{\left\langle\theta_{Q}^{(i)}, \varphi_{P}\right\rangle}\left\langle\vec{f}, \varphi_{P}\right\rangle=\sum_{P} a_{Q P}^{(i)} \vec{s}_{P}
$$

Since supp $\hat{\varphi}_{P} \cap \operatorname{supp} \hat{\theta}_{Q}^{(i)} \neq\{\emptyset\}$ only if $l(Q)=2^{j} l(P)$ with $j=1,2,3,4$ (recall that supp $\hat{\varphi}_{P} \subseteq\left\{\xi \in \mathbb{R}^{n}: 2^{\mu-1} \leq|\xi| \leq 2^{\mu+1}\right\}$ when $\left.l(P)=2^{-\mu}\right)$, we see that $a_{Q P}^{(i)}=\left\langle\theta_{Q}^{(i)}, \varphi_{P}\right\rangle=0$ unless $2 \leq \frac{l(Q)}{l(P)} \leq 16$, in which case

$$
\left|a_{Q P}\right| \leq c_{M}\left(1+\frac{\left|x_{Q}-x_{P}\right|}{l(Q)}\right)^{-M} \text { for each } M>0,
$$

as was shown in 3], p. 72. Let $M>\frac{n}{p^{\prime}}+\frac{\beta}{p}$. Then $A^{(i)}:=\left(a_{Q P}^{(i)}\right)$ is an almost diagonal matrix for each $i$, and, by Theorem 1.10

$$
\left\|\left\{\vec{t}_{Q}^{(i)}\right\}_{Q}\right\|_{\dot{b}_{p}^{\alpha q}(W)} \leq c\left\|\left\{\vec{s}_{Q}\right\}_{Q}\right\|_{\dot{b}_{p}^{\alpha q}(W)} .
$$

Combining (10.3) with (10.2), we get the opposite direction of (10.1).

Corollary 10.3. Let $\left\{{ }_{N} \psi^{(i)}\right\}, i=1, \ldots, 2^{n}-1$, be a collection of Daubechies DN generating wavelet functions for $L^{2}\left(\mathbb{R}^{n}\right)$ with compact supports linearly dependent on $N$ (for more details, see [2]). Then for any $\vec{f}$ with $f_{j} \in \mathcal{S}^{\prime} / \mathcal{P}\left(\mathbb{R}^{n}\right), j=1, \ldots, m$,

$$
\|\vec{f}\|_{\dot{B}_{p}^{\alpha q}(W)} \approx \sum_{i=1}^{2^{n}-1}\left\|\left\{\left\langle\vec{f},{ }_{N} \psi_{Q}^{(i)}\right\rangle\right\}_{Q}\right\|_{b_{p}^{\alpha q}(W)}
$$

for sufficiently large $N$.

Proof. First, observe that there exists a constant $c$ such that for all $i=1, \ldots, 2^{n}-$ 1, the functions $\frac{N \psi^{(i)}}{c}$ are smooth molecules, and so $\left\{\frac{{ }_{N} \psi_{Q}^{(i)}}{c}\right\}_{Q}$ is a family of smooth molecules for $\dot{B}_{p}^{\alpha q}(W)$ if we choose $N$ sufficiently large to have the necessary smoothness and vanishing moments. Second, if $\varphi \in \mathcal{A}$, then $\left(\left\langle{ }_{N} \psi_{Q}^{(i)}, \varphi_{P}\right\rangle_{Q P}\right) \in$ $\operatorname{ad}_{p}^{\alpha q}(\beta)$ by (8.2). Applying these two facts in the proof of the previous theorem, we get (10.4).

\section{Inhomogeneous Besov SPACES}

In this section we discuss the inhomogeneous spaces. Before we define the vectorvalued inhomogeneous Besov space $B_{p}^{\alpha q}(W)$ with matrix weight $W$, we introduce a class of functions $\mathcal{A}^{(I)}$ with properties similar to those of an admissible kernel: we say $\Phi \in \mathcal{A}^{(I)}$ if $\Phi \in \mathcal{S}\left(\mathbb{R}^{n}\right)$, supp $\hat{\Phi} \subseteq\left\{\xi \in \mathbb{R}^{n}:|\xi| \leq 2\right\}$ and $|\hat{\Phi}(\xi)| \geq c>0$ if $|\xi| \leq$ $\frac{5}{3}$. 
Definition 11.1 (Inhomogeneous matrix-weighted Besov space $B_{p}^{\alpha q}(W)$ ). For $\quad \alpha$ $\in \mathbb{R}, 1 \leq p<\infty, 0<q \leq \infty, W$ a matrix weight, $\varphi \in \mathcal{A}$ and $\Phi \in \mathcal{A}^{(I)}$, we define the Besov space $B_{p}^{\alpha q}(W)$ as the collection of all vector-valued distributions $\vec{f}=\left(f_{1}, \ldots, f_{m}\right)^{\mathrm{T}}$ with $f_{i} \in \mathcal{S}^{\prime}\left(\mathbb{R}^{n}\right), 1 \leq i \leq m$, such that

$$
\|\vec{f}\|_{B_{p}^{\alpha q}(W)}=\|\Phi * \vec{f}\|_{L^{p}(W)}+\left\|\left\{2^{\nu \alpha}\left\|\varphi_{\nu} * \vec{f}\right\|_{L^{p}(W)}\right\}_{\nu \geq 0}\right\|_{l^{q}}<\infty,
$$

where the $l^{q}$-norm is replaced by the supremum on $\nu \geq 1$ if $q=\infty$.

Note that now we consider all vector-valued distributions in $\mathcal{S}^{\prime}\left(\mathbb{R}^{n}\right)$ (rather than $\mathcal{S}^{\prime} / \mathcal{P}$ as in the homogeneous case), since $\hat{\Phi}(0) \neq 0$.

The corresponding inhomogeneous weighted sequence Besov space $b_{p}^{\alpha q}(W)$ is defined for the vector sequences enumerated by the dyadic cubes $Q$ with $l(Q) \leq 1$.

Definition 11.2 (Inhomogeneous weighted sequence Besov space $b_{p}^{\alpha q}(W)$ ). For $\alpha$ $\in \mathbb{R}, 1 \leq p<\infty, 0<q \leq \infty$, and $W$ a matrix weight, the space $b_{p}^{\alpha q}(W)$ consists of all vector-valued sequences $\vec{s}=\left\{\vec{s}_{Q}\right\}_{l(Q) \leq 1}$ such that

$$
\|\vec{s}\|_{b_{p}^{\alpha q}(W)}=\left\|\left\{2^{\nu \alpha}\left\|\sum_{l(Q)=2^{-\nu}}|Q|^{-\frac{1}{2}} \vec{s}_{Q} \chi_{Q}\right\|_{L^{p}(W)}\right\}_{\nu \geq 0}\right\|_{l^{q}}<\infty,
$$

where the $l^{q}$-norm is again replaced by the supremum on $\nu \geq 1$ if $q=\infty$.

Following [5], given $\varphi \in \mathcal{A}$ and $\Phi \in \mathcal{A}^{(I)}$, we select $\psi \in \mathcal{A}$ and $\Psi \in \mathcal{A}^{(I)}$ such that

$$
\hat{\tilde{\Phi}}(\xi) \cdot \hat{\Psi}(\xi)+\sum_{\nu \geq 1} \hat{\tilde{\varphi}}\left(2^{-\nu} \xi\right) \cdot \hat{\psi}\left(2^{-\nu} \xi\right)=1 \text { for all } \xi
$$

where $\tilde{\Phi}(x)=\overline{\Phi(-x)}$. Analogously to the $\varphi$-transform decomposition (2.2), we have the identity for $f \in \mathcal{S}^{\prime}\left(\mathbb{R}^{n}\right)$ :

$$
f=\sum_{l(Q)=1}\left\langle f, \Phi_{Q}\right\rangle \Psi_{Q}+\sum_{\nu=1}^{\infty} \sum_{l(Q)=2^{-\nu}}\left\langle f, \varphi_{Q}\right\rangle \psi_{Q}
$$

where $\Phi_{Q}(x)=|Q|^{-1 / 2} \Phi\left(2^{\nu} x-k\right)$ for $Q=Q_{\nu k}$, and $\Psi_{Q}$ is defined similarly.

For each $\vec{f}$ with $f_{i} \in \mathcal{S}^{\prime}\left(\mathbb{R}^{n}\right)$, we define the inhomogeneous $\varphi$-transform $S_{\varphi}^{(I)}$ : $B_{p}^{\alpha q}(W) \longrightarrow b_{p}^{\alpha q}(W)$ by setting $\left(S_{\varphi}^{(I)} \vec{f}\right)_{Q}=\left\langle\vec{f}, \varphi_{Q}\right\rangle$ if $l(Q)<1$, and $\left(S_{\varphi}^{(I)} \vec{f}\right)_{Q}=$ $\left\langle\vec{f}, \Phi_{Q}\right\rangle$ if $l(Q)=1$.

The inverse inhomogeneous $\varphi$-transform $T_{\psi}^{(I)}$ is the map taking a sequence $s=$ $\left\{s_{Q}\right\}_{l(Q) \leq 1}$ to $T_{\psi}^{(I)} s=\sum_{l(Q)=1} s_{Q} \Psi_{Q}+\sum_{l(Q)<1} s_{Q} \psi_{Q}$. In the vector case, $T_{\psi}^{(I)} \vec{s}=$ $\sum_{l(Q)=1} \vec{s}_{Q} \Psi_{Q}+\sum_{l(Q)<1} \vec{s}_{Q} \psi_{Q}$. By (11.2), $T_{\psi}^{(I)} \circ S_{\varphi}^{(I)}$ is the identity on $\mathcal{S}^{\prime}\left(\mathbb{R}^{n}\right)$.

Next we show that the relation between $B_{p}^{\alpha q}(W)$ and $b_{p}^{\alpha q}(W)$ is the same as for the homogeneous spaces. 
Theorem 11.3. Let $\alpha \in \mathbb{R}, 0<q \leq \infty, 1<p<\infty$, and let $W$ satisfy any of (A1)-(A3). Then

$$
\|\vec{f}\|_{B_{p}^{\alpha q}(W)} \approx\left\|\left\{\vec{s}_{Q}(\vec{f})\right\}_{l(Q) \leq 1}\right\|_{b_{p}^{\alpha q}(W)} .
$$

Before we outline the proof, we need to adjust the notation of smooth molecules for the inhomogeneous case. Define a family of smooth molecules $\left\{m_{Q}\right\}_{l(Q) \leq 1}$ for $B_{p}^{\alpha q}(W)$ as a collection of functions with the properties:

1. for dyadic $Q$ with $l(Q)<1$, each $m_{Q}$ is a smooth $(\delta, M, N)$-molecule with (M.i)-(M.iii) as for the homogeneous space $\dot{B}_{p}^{\alpha q}(W)$ (see Section 5);

2. for dyadic $Q$ with $l(Q)=1$, each $m_{Q}$ (sometimes we denote it as $M_{Q}$ to emphasize the difference) satisfies (M3), (M4) and a modification of (M2) (which makes it a particular case of (M3) when $\gamma=0$ ):

$$
\left|m_{Q}(x)\right| \leq|Q|^{-1 / 2}\left(1+\frac{\left|x-x_{Q}\right|}{l(Q)}\right)^{-M} .
$$

Note that $M_{Q}$ does not necessarily have vanishing moments. Now one direction of the norm equivalence (11.3) comes from the modified version of Theorem 5.2 .

Theorem 11.4. Let $\alpha \in \mathbb{R}, 1 \leq p<\infty, 0<q \leq \infty$ and $W$ be a doubling matrix weight of order $p$. Suppose $\left\{m_{Q}\right\}_{l(Q) \leq 1}$ is a family of smooth molecules for $B_{p}^{\alpha q}(W)$. Then

$$
\left\|\sum_{l(Q) \leq 1} \vec{s}_{Q} m_{Q}\right\|_{B_{p}^{\alpha q}(W)} \leq c\left\|\left\{\vec{s}_{Q}\right\}_{l(Q) \leq 1}\right\|_{b_{p}^{\alpha q}(W)} .
$$

Sketch of the Proof. We have

$$
\begin{aligned}
& \left\|\sum_{l(Q) \leq 1} \vec{s}_{Q} m_{Q}\right\|_{B_{p}^{\alpha q}(W)}=\left\|\sum_{l(Q) \leq 1} \vec{s}_{Q}\left(\Phi * m_{Q}\right)\right\|_{L^{p}(W)} \\
& +\left\|\left\{2^{\nu \alpha}\left\|\sum_{l(Q) \leq 1} \vec{s}_{Q}\left(\varphi_{\nu} * m_{Q}\right)\right\|_{L^{p}(W)}\right\}_{\nu \geq 1}\right\|_{l^{q}}=I+I I .
\end{aligned}
$$

As in Theorem [5.2 which uses the convolution estimates (5.2) and (5.3), we need similar inequalities for modified molecules (the proofs are routine applications of Lemmas B.1 and B.2 from [5]):

$$
\begin{gathered}
\left|\Phi * M_{Q}(x)\right| \leq c\left(1+\left|x-x_{Q}\right|\right)^{-M} \text { when } l(Q)=1, \\
\left|\Phi * m_{Q}(x)\right| \leq c|Q|^{-\frac{1}{2}} 2^{-\mu \sigma}\left(1+\left|x-x_{Q}\right|\right)^{-M} \text { for some } \sigma>J-\alpha
\end{gathered}
$$

when $l(Q)=2^{-\mu}, \mu \geq 1$, and

$$
\left|\varphi_{\nu} * M_{Q}(x)\right| \leq c 2^{-\nu \tau}\left(1+\left|x-x_{Q}\right|\right)^{-M} \text { for some } \tau>\alpha
$$

when $\nu \geq 1$ and $l(Q)=1$. For $\nu \geq 1$ and $l(Q)<1$, the estimate of $\left|\left(\varphi_{\nu} * m_{Q}\right)(x)\right|$ comes from either (5.2) or (5.3). 
To estimate $I$ we use (11.5) and (11.6) (note that (11.5) is a special case of (11.6) for $\mu=0$ ) and follow the steps of Theorem 1.10 by using Hölder's inequality twice to bring the $p^{\text {th }}$ power inside of the sum, and the Squeeze and the Summation Lemmas from Section 5 (it is essential that $\sigma>J-\alpha$ for convergence purposes) to get

$$
I \leq c\left\|\left\{\vec{s}_{Q}\right\}_{l(Q) \leq 1}\right\|_{b_{p}^{\alpha q}(W)}
$$

The second term $I I$ is also estimated by $\left\|\left\{\vec{s}_{Q}\right\}_{l(Q) \leq 1}\right\|_{b_{p}^{\alpha q}(W)}$, which is obtained by exact repetition of the proof of Theorem 5.2 , only restricting the sum over $\mu \in \mathbb{Z}$ to the sum over $\mu \geq 0$. Also note that (111.7) is a particular case of (5.3) when $\mu=0$ and, thus, $l(Q)=1$. Therefore, (11.4) is proved.

In particular, since $\Phi$ and $\Psi$ generate families of smooth molecules for $B_{p}^{\alpha q}(W)$, we get

$$
\|\vec{f}\|_{B_{p}^{\alpha q}(W)} \leq c\left\|\left\{\vec{s}_{Q}(\vec{f})\right\}_{l(Q) \leq 1}\right\|_{b_{p}^{\alpha q}(W)},
$$

which gives one direction of the norm equivalence (11.3). To show the other direction, i.e., that the (inhomogeneous) $\varphi$-transform is bounded, we simply observe that $\tilde{\Phi} * \vec{f} \in E_{0}$, which is true since $(\tilde{\Phi} * \vec{f})_{i} \in \mathcal{S}^{\prime}$ and $\operatorname{supp} \hat{\tilde{\Phi}} \subseteq\left\{\xi \in \mathbb{R}^{n}:|\xi| \leq 2\right\}$. Hence, Lemmas 6.3 and 6.5 apply to $\vec{g}=\Phi * \vec{f}$ as stated. We have

$$
\begin{gathered}
\left\|\left\{\vec{s}_{Q}(\vec{f})\right\}_{l(Q) \leq 1}\right\|_{b_{p}^{\alpha q}(W)} \approx\left\|\sum_{k \in \mathbb{Z}^{n}}(\tilde{\Phi} * \vec{f})(k) \chi_{Q_{0 k}}\right\|_{L^{p}(W)} \\
+\left\|\left\{2^{\nu \alpha}\left\|\sum_{l(Q)=2^{-\nu}}|Q|^{-\frac{1}{2}} \chi_{Q}\left\langle\vec{f}, \varphi_{Q}\right\rangle\right\|_{L^{p}(W)}\right\}_{\nu \geq 1}\right\|_{l^{q}}=I+I I .
\end{gathered}
$$

Using $\tilde{\Phi} * \vec{f} \in E_{0}$ and repeating the proof of Theorem 6.6 for both terms (in the second term we take the $l^{q}$ norm only over $\nu \in \mathbb{N}$ ), we get the desired estimate:

$$
\left\|\left\{\vec{s}_{Q}(\vec{f})\right\}_{l(Q) \leq 1}\right\|_{b_{p}^{\alpha q}(W)} \leq c\|\vec{f}\|_{B_{p}^{\alpha q}(W)} .
$$

Note that as a consequence we also get independence of $B_{p}^{\alpha q}(W)$ from the choices of $\Phi$ and $\varphi$.

Now we will briefly discuss operators on the inhomogeneous spaces. An almost diagonal matrix on $b_{p}^{\alpha q}(W)$ is the matrix $A=\left(a_{Q P}\right)_{l(Q), l(P) \leq 1}$ whose entries satisfy (8.1), i.e., $\left|a_{Q P}\right|$ is bounded by (8.1) only for dyadic $Q, \bar{P}$ with $l(Q), l(P) \leq 1$. Such a matrix $A$ is a bounded operator on $b_{p}^{\alpha q}(W)$ for the following reasons: let $\vec{s} \in b_{p}^{\alpha q}(W)$ and then define $\overrightarrow{\dot{s}}=\left\{\overrightarrow{\dot{s}}_{Q}\right\}_{Q \text { dyadic }}$ by setting $\overrightarrow{\dot{s}}_{Q}=\vec{s}_{Q}$ if $l(Q) \leq 1$ and $\overrightarrow{\dot{s}}_{Q}=0$ if $l(Q)>1$. Note that $\vec{s}$ is a restriction of $\overrightarrow{\dot{s}}$ on $b_{p}^{\alpha q}(W)$. Also, set $\dot{A}=\left(\dot{a}_{Q P}\right)_{Q, P \text { dyadic }}$, putting $\dot{a}_{Q P}=a_{Q P}$ if $l(Q), l(P) \leq 1$ and $\dot{a}_{Q P}=0$ otherwise. 
Then

$$
\begin{aligned}
\|A \vec{s}\|_{b_{p}^{\alpha q}(W)} & =\left\|\left\{\sum_{l(P) \leq 1} a_{Q P} \vec{s}_{P}\right\}_{l(Q) \leq 1}\right\|_{b_{p}^{\alpha q}(W)} \\
& =\left\|\left\{\sum_{P \text { dyadic }} \dot{a}_{Q P} \overrightarrow{\vec{s}}_{P}\right\}_{Q}\right\|_{\dot{b}_{p}^{\alpha q}(W)} \leq c\|\vec{s}\|_{\dot{b}_{p}^{\alpha q}(W)},
\end{aligned}
$$

by Theorem 1.10] By the construction, $\|\vec{s}\|_{\dot{b}_{p}^{\alpha q}(W)}=\|\vec{s}\|_{b_{p}^{\alpha q}(W)}$, and so we get boundedness of $A$ on $b_{p}^{\alpha q}(W)$.

It is easy to see that the class of almost diagonal matrices on $b_{p}^{\alpha q}(W)$ is closed under composition. The same statements (boundedness and being closed under composition) are true for the corresponding almost diagonal operators on $B_{p}^{\alpha q}(W)$ by combining the norm equivalence (11.3) and the above results about almost diagonal matrices on $b_{p}^{\alpha q}(W)$. For Calderón-Zygmund operators on inhomogeneous matrix-weighted Besov spaces, some minor notational changes should be made. The collection of smooth $N$-atoms $\left\{a_{Q}\right\}_{Q \text { dyadic }}$ in the homogeneous case ought to be replaced by the set of atoms $\left\{a_{Q}\right\}_{l(Q)<1} \cup\left\{A_{Q}\right\}_{l(Q)=1}$, where the $a_{Q}$ 's have the same properties as before and the $A_{Q}$ 's are such that supp $A_{Q} \subseteq 3 Q$ and $\left|D^{\gamma} A_{Q}(x)\right| \leq 1$ for $\gamma \in \mathbb{Z}_{+}^{n}$. This leads to a slight change of the smooth atomic decomposition (see [5], p. 132):

$$
f=\sum_{l(Q)<1} s_{Q} a_{Q}+\sum_{l(Q)=1} s_{Q} A_{Q}
$$

With these adjustments, all corresponding statements about CZOs hold with essentially the same formulations for the inhomogeneous spaces. Thus, all results obtained for the matrix-weighted homogeneous Besov spaces are essentially the same for the inhomogeneous case.

\section{ACKNowledgements}

The research presented in this paper is a part of the author's Ph.D. thesis directed by Prof. Michael Frazier. The author would like to express her gratitude to Prof. Frazier for introducing the subject matter, for valuable discussions in the course of this work, and for many useful comments and suggestions while examining the paper. She would also like to thank Prof. Fedor Nazarov and Prof. Alexander Volberg for helpful discussions.

\section{REFERENCES}

1. M.Christ And M.Goldberg, Vector $A_{2}$ weights and a Hardy-Littlewood maximal function, Trans. Amer. Math. Soc., 353, no. 5, (2001), 1995-2002.

2. I.Daubechies, Orthonormal bases of compactly supported wavelets, Comm. Pure Appl. Math. 41 (1988), 909-996. MR 90m:42039

3. M.Frazier, B.Jawerth And G.Weiss, Littlewood-Paley Theory and Study of Function Spaces, CBMS Regional Conference Series in Mathematics 79, Amer. Math. Soc., Providence, RI (1991). MR 92m:42021

4. M.Frazier and B.Jawerth, Decomposition of Besov Spaces, Indiana Univ. Math. J. 34 (1985), 777-799. MR 87b:46083

5. M.Frazier and B.JaWerth, A discrete transform and decompositions of distribution spaces, J. Funct. Anal. 93 (1990), 34-170. MR 92a:46042 
6. M.Frazier, R.Torres And G.Weiss, The boundedness of Calderón-Zygmund operators on the spaces $\dot{F}_{p}^{\alpha q}(W)$, Rev. Mat. Iberoamericana 4, no.1, (1988), 41-72. MR 90k:42029

7. D.Goldberg, A local version of real Hardy spaces, Duke Math. J. 46 (1979), 27-42. MR 80h:46052

8. R.Hunt, B.Muckenhoupt and R.Wheeden, Weighted norm inequalities for conjugate function and Hilbert transform, Trans. Amer. Math. Soc., 176 (1973), 227-251. MR 47:701

9. P.G.Lemarié And Y.Meyer, Ondelettes et bases hilbertiennes, Rev. Mat. Iberoamericana 2 (1986), 1-18.

10. Y.MeYER, Principe d'incertitude, bases Hilbertiennes et algèbres d'opérateurs, Séminaire Bourbaki 1985/86, Exposé 662, Astérisque, no. 145-146, Soc. Math. France, Paris, 1987, pp. 209-223. MR 88g:42012

11. Y.MeYer, Wavelets and Operators, Cambridge University Press, 1992. MR 92f:42001

12. F.NAZARov And S.Treil, The hunt for a Bellman function: applications to estimates for singular integral operators and to other classical problems of harmonic analysis, Algebra i Analiz 8 (1996), no. 5, 32-162; English transl., St. Petersburg Math. J. 8 (1997), 721-824. MR 99d:42026

13. S.Roudenko, The theory of function spaces with matrix weights, Ph.D. thesis, Michigan State Univ., 2002.

14. E.Stein, Harmonic Analysis, Princeton University Press, 1993. MR 95c: 42002

15. H.Triebel, Theory of function spaces, Monographs in Math., vol. 78, Birkhäuser-Verlag, Basel, 1983. MR 86j:46026

16. S.Treil And A.VolberG, Wavelets and the angle between past and future, J. Funct. Anal. 143 (1997), no.2, 269-308. MR 99k:42073

17. A.Volberg, Matrix $A_{p}$ weights via S-functions, J. Amer. Math. Soc. 10 (1997), no.2, 445-466. MR 98a:42013

Department of Mathematics, Michigan State University, East Lansing, Michigan 48824

E-mail address: svetlana@math.msu.edu

Current address: Department of Mathematics, Duke University, Box 90320, Durham, North Carolina 27708 